Historic, Archive Document

Do not assume content reflects current scientific knowledge, policies, or practices. 

FOREST SURVEY RELEASE NO. 53

\subsection{2 \\ A $3 F 76$}

$+$

\section{$1 \div 04+19060$}

WNIE StTY OF CAMGSRIA

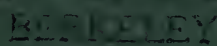

$c x=-\ldots+1$

MOY 1 is 1950

1957 PULPWOOD PRODUCTION

IN THE SOUTH

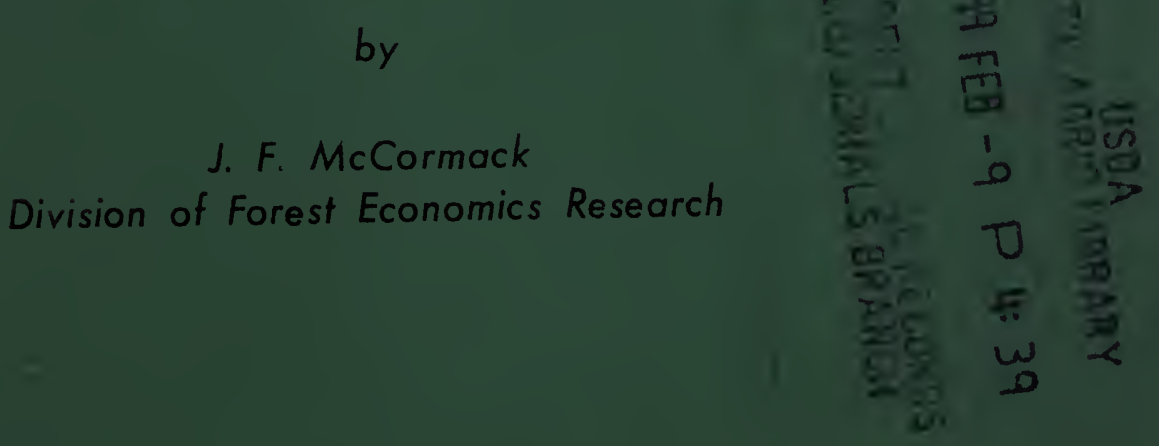

U.S. DEPARTMENT OF AGRICULTURE FOREST SERVICE

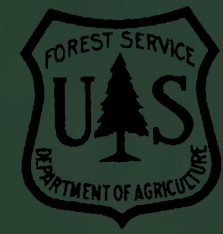

SOUTHEASTERN FOREST EXPERIMENT STATION

ASHEVILLE, N. C.

and

SOUTHERN FOREST EXPERIMENT STATION

NEW ORLEANS, LA.

in cooperation with

SOUTHERN PULPWOOD CONSERVATION ASSOCIATION

ATLANTA, GA. 


\section{PREFACE}

Trrough the McSweeney-McNary Act of 1928, Congress author1zed the Secretary of Agriculture to conduct a survey of the forest resources of the United States. The Forest Survey was organized by the Forest Service to carry out the provisions of the Act. The Southeastern Forest Experiment Station, Asheville, North Carolina, is responsible for the work in Virginia, North Carolina, South Carolina, Georgia, and Florida. The Southern Forest Experiment Station, New Orleans, Louisiana, directs the work in Alabama, Arkansas, Louisiana, Mississippi, Oklahoma, Tennessee, and Texas.

The work of the Survey is divided into five major phases:

1. Inventory. Determination of the extent, location, and condition of forest lands, and the quantity, species, and quality of timber on these lands.

2. Growth and mortality. Determination of the current rate of timber growth, and the total loss resulting from fire, insects, disease, suppression, and other causes.

3. Timber cut. Determination of the amount of industrial and domestic wood used, and the amount removed by landclearing and cultural operations.

4. Requirements. Determination of the current and probable future requirements for forest products by all classes of consumers.

5. Policies and plans. Analysis of the relation of these findings to one another and to other economic factors as a basis for public and private policies and plans of forest land use and management.

\section{ACKNOWLEDGMENT}

The statistics presented herein are based upon reports furnished by the rood vrocurement officlals representing 76 mills located in or drawing wood from the soutk. Reports showed production either by county w by shipping points that were used to assign production to counties. Conty data are approxiaste, hut totals for small groups of counties ebroll have little error. 
1957 PULPWOOD PRODUCTION IN THE SOUTH

(Forest Survey Release No. 53)

\section{PLEASE NOTE:}

Numbers used in figure 2 are in thousands of cords. 



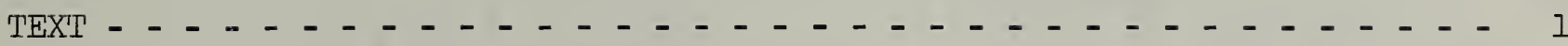

FIGURES:

1. Pulpmills drawing wood from the South, 1957 - - - - - - - - ii

2. Pulpwood production in the South, 1957 - - - . - - - - - 3

3. Pine pulpwood production by county in the South, 1957 . . - . - 16

4. Hardwood pulpwood production by county in the South, 1957 - _ - - 17

\section{TABLES:}

1. Round pulpwood production in the South by state and species group,

1956 and 1957 . . . . . . . . . . . . . . . . 1

2. Wood residues produced for pulp manufacture by state, type, and species, 1957 - . . . . . . . . . . . . . 2

3. Pulpwood production in Southeastern Station territory by State and Survey Uni.t, 1957 - - - - - - - - - - - - - - - - 4

4. Pulpwood production in Southern Station territory by State and Survey Unit, 1957 - . . . . . . . . . . . . . . - 4 ROUND PULPWOOD PRODUCTION, 1957:

5. In Alabama _ . . . . . . . . . . . . . . . . 5

6. In Arkansas _ - . . . . . . . . . . . . . 5

7. In Florida - _ . . . . . . . . . . . . . . 6

8. In Georgia - - . - . . . . . . . . . . - 7

9. In Louisiana - - . - . - . . . . . . . - 8

10. In Mississippi - - - - - - - . - - . - . - 9

11. In North Carolina - - - - - . - . - - - . - 10

12. In Oklahoma - . . . . . . . . . . . . . . 10

13. In South Carolina _ . . . . . . . . . . . . . 11

14. In Tennessee - . . . . . . . . . . . . . . . 11

15. In Texas - . . . . . . . . . . . . . . 12

16. In Virginia - . . . . . . . . . . . . . . 13

17. Companies drawing pulpwood from the South, 1957 - . - - - - - 14

18. Pulpmills under construction or announced in the South - _ - . - - 15 


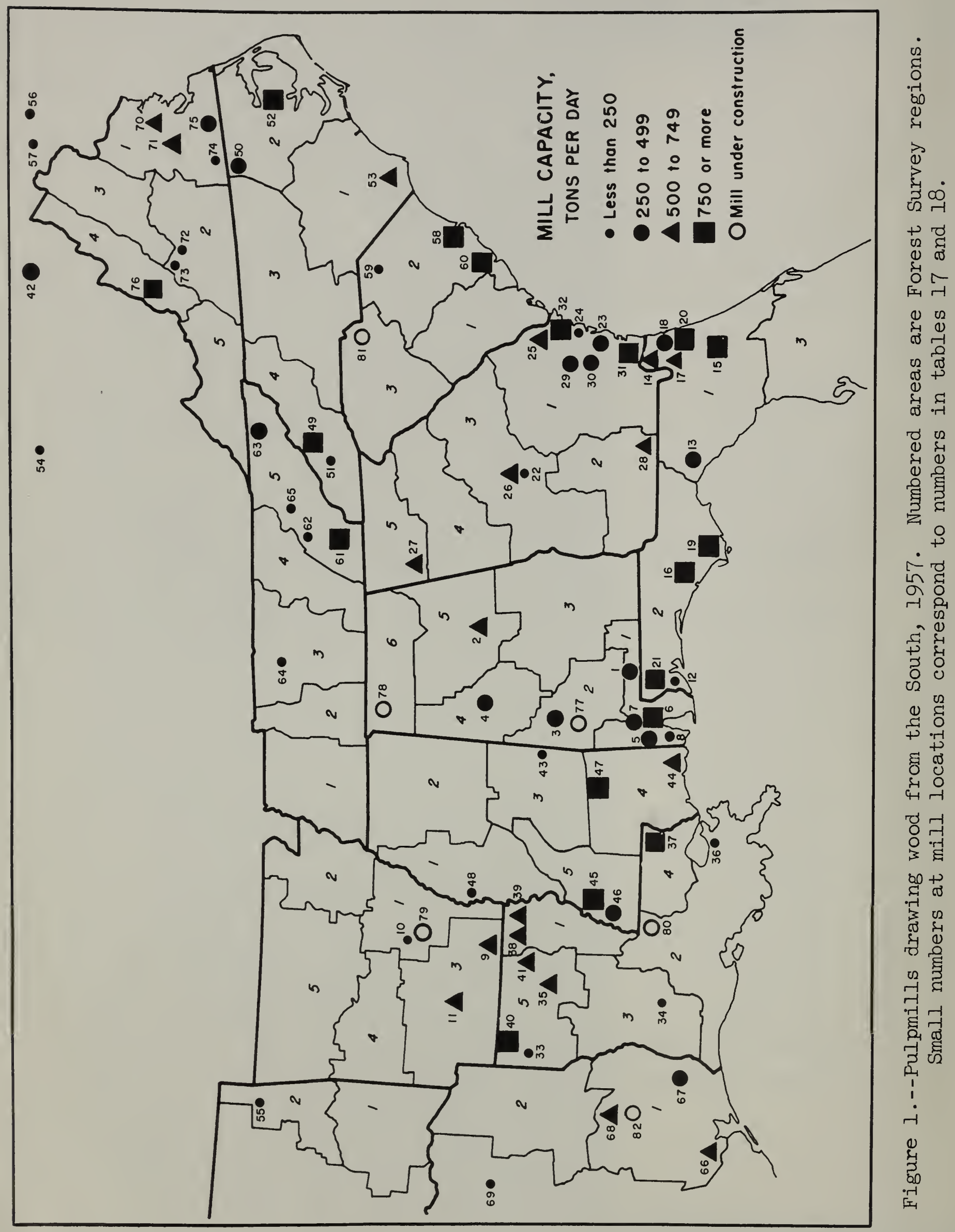




\section{PUIPWOOD PRODUCTION IN THE SOUTH}

\section{J. F. McCormack}

\section{Southeastern Forest Experiment Station}

Pulpwood production in southern states during 1957 fell slightly below the 1956 level, thus reversing a strong, long-term upward trend. Total production amounted to $19,782,600$ cords, a decrease of 562,300 cords, or 2.8 percent, from the 1956 record high. At the same time, the combined pulping capacity of southern mills climbed from 38,000 tons per day in 1956 to 41,000 tons in 1957 as new mills and expanded productive equipment in existing mills went into operation.

The decline in 1957 production is associated with the current business recession. Similar trend effects were experienced in the recession years 1954 and 1949. As general business conditions improve, the amount of pulpwood used annually in the South can be expected to resume its upward trend.

Round pulpwood accounted for $18,579,600$ cords, or 94 percent, of the total amount produced, and wood residues obtained from other manufacturing processes made up the remaining 1,203,000 cords. As indicated in Table 1, the production of roundwood was 5.6 percent below 1956, with all states except Alabama showing a decrease. The decline by states ranged from a slight decrease in Florida to a 20-percent drop in Oklahoma. The decline in roundwood production was balanced to some extent by increased use of wood residues.

Table 1.--Round pulpwood production in the South by State and species group, 1956 and 1957

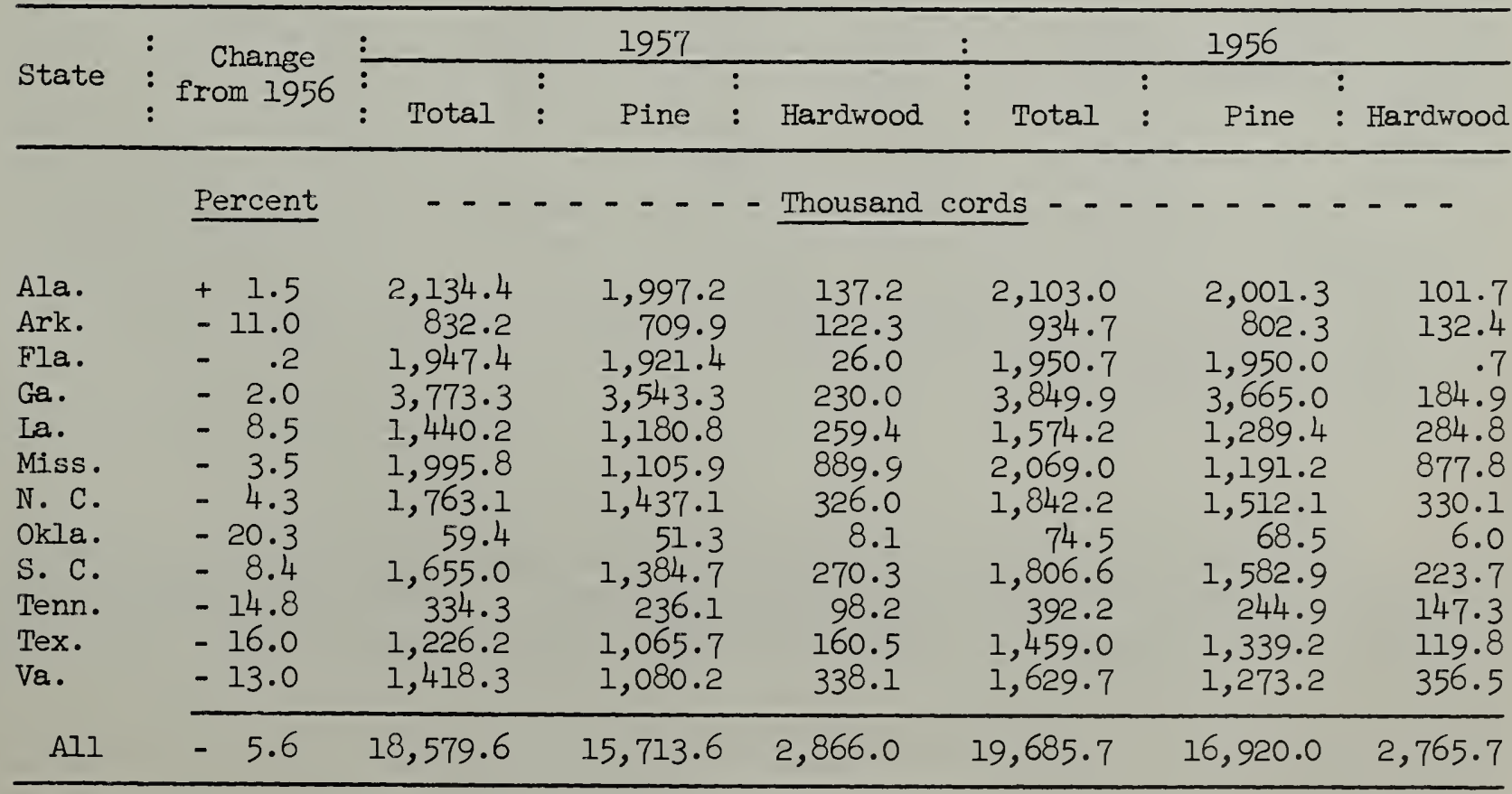


Two trends are evident which are very favorable from the standpoint of timber supply and more efflcient timber utilization. In recent years, the proportion of wood coming from hardwood specles and from wood residues have both been increasing. Hardwood species, which made up 14.0 percent of the roundwood produced in 1956, accounted for 15.4 percent in 1957, a gain of about 100,000 cords. The increasing use of hardwoods in pulp manufacture has exhibited a slow but steady gain for the past several years. This is a desirable trend, because it shifts more of the production from the heavily used pine species to the more abundant hardwoods.

The use of coarse, chippable residues obtained from sawmills, veneer plants, and other sources has shown a much more striking increase. Chipping of this type of material has risen from 76,000 cords in 1953 to 1.2 million cords in 1957 (table 2). The average rate of use has doubled each year, and residues now comprise 6 percent of the total pulpwood production in the South. Production in 1957 was 83 percent above 1956, and it can be expected to go higher.

Table 2.--Wood residues produced for pulp manufacture by state, type, and species, 1957

(In thousand cords)

\begin{tabular}{|c|c|c|c|c|c|c|c|}
\hline \multirow{2}{*}{ State } & \multirow{2}{*}{ : $\quad \begin{array}{l}\text { AlI } \\
: \text { types }\end{array}$} & \multicolumn{3}{|c|}{ Sawmill chips } & \multicolumn{3}{|c|}{$: \quad$ Other residues I/ } \\
\hline & & Total & : Pine & : Hardwood & : Total & : Pine & : Hardwood \\
\hline Alabama & 159.8 & 148.3 & 133.4 & 14.9 & 11.5 & 3.0 & 8.5 \\
\hline Arkansas & 210.2 & 207.8 & 205.4 & $2 . \overline{4}$ & 2.5 & .8 & 1.6 \\
\hline Florida & 50.1 & 48.5 & 48.1 & .4 & 1.6 & .8 & .8 \\
\hline Georgia & 85.7 & 74.9 & 69.0 & 5.9 & 10.8 & 1.4 & 9.4 \\
\hline Louisiana & 124.0 & 122.0 & 113.0 & 9.0 & 2.0 & .8 & 1.2 \\
\hline Mississippi & 79.9 & 62.0 & 28.1 & 33.9 & $17 \cdot 9$ & .3 & 17.6 \\
\hline North Carolina & 102.7 & 86.9 & 82.8 & 4.1 & 15.8 & 2.5 & $13 \cdot 3$ \\
\hline South Carolina & 88.0 & $77 \cdot 9$ & 76.2 & 1.7 & 10.1 & .6 & 9.5 \\
\hline Tennessee & 6.9 & 5.0 & -- & 5.0 & 1.9 & -- & 1.9 \\
\hline Texas?] & 195.6 & 195.1 & 194.7 & .4 & .5 & .5 & - \\
\hline Virginia & 100.1 & 97.0 & $97 \cdot 0$ & -- & $3 \cdot 1$ & -- & 3.1 \\
\hline All states & $1,203.0$ & $1,125.4$ & $1,047.7$ & $77 \cdot 7$ & 77.6 & 10.7 & 66.9 \\
\hline
\end{tabular}

I) Veneer cores, pole and piling trim, cull crossties, etc.

2) Includes Oklahoma.

Production of chips from sawmill slabs and trim has been the basis for most of the increased use of wood residues. A number of sawmills have installed log debarkers to remove the bark before logs are sawn. The debarked 
slabs and edgings are then chipped and sent to the pulpmill. In other cases, slabs and trim are being collected from portable sawmill sites and concentrated at yards where the debarking and chipping are done. The volume of sawmill and veneer mill chips received at pulpmills accounted for 94 percent of all residues used, and pine residues made up 88 percent of the total.

The State of Georgia retained its lead in pulpwood production during 1957 by producing a total of 3.9 million cords (fig.2). Louisiana displaced Virginia as seventh of the twelve southern states in total volume of wood produced. The State of Arkansas led in production of wood residues. Eight individual counties, located in four different states, each produced over 100,000 cords of wood. The

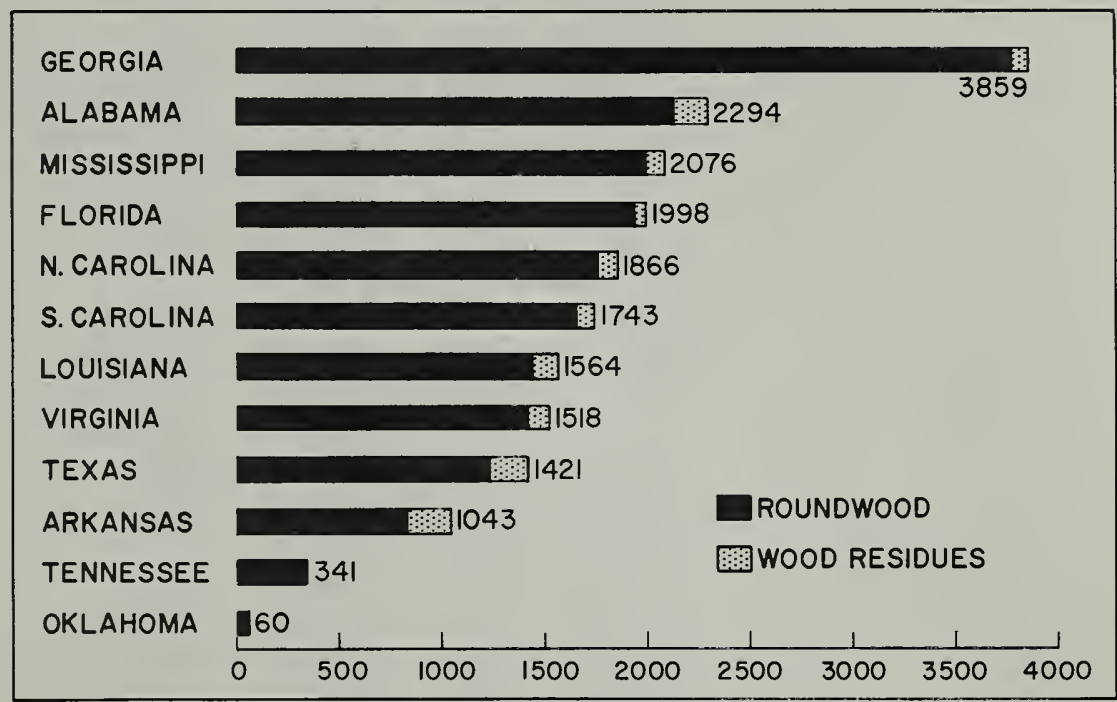

Fig. 2.--Pulpwood production in the South, 1957. Roundwood and residues combined.

leader was Baldwin

County, Alabama, with a volume of 169,500 cords. Production of pine pulpwood was concentrated in southeast Georgia and northeast Florida (fig. 3 ). These two areas furnished 17 percent of the round pine wood cut in the South. A similar map for hardwood pulpwood ( $f i g .4$ ) shows concentrations of production in southern Mississippi, western North Carolina, and the central. Mountain Region of Virginia.

The number of pulpmills operating within the twelve southern states during 1957 increased to 70, as two new mills went into operation in Alabama. These were the Container Corporation of America mill at Brewton, and the Gulf States Paper Corporation mill at Demopolis. Their combined production added 600 tons per day to the capacity of the industry. In January 1958 two other new mills went into production. They were the Dierks Paper Company mill at Pine Bluff, Arkansas, and the Rayonier mill at Jesup, Georgia. Plans for still another new mill were announced by the Tennessee River Pulp and Paper Company. The site will be at Sheffield, Alabama. The Mead Corporation mill at Nashville, Tennessee, was shut down in March 1958 because of economic conditions in the industry. 
Table 3.--Pulpwood production in Southeastern Station territory by State and Survey unit, 1957

\begin{tabular}{|c|c|c|c|c|}
\hline \multirow{2}{*}{ State } & \multirow{2}{*}{$\begin{array}{l}\text { Forest } \\
\text { Survey, } \\
\text { unitIl }\end{array}$} & \multicolumn{2}{|c|}{ Species group } & \multirow{2}{*}{$\begin{array}{l}: \\
-:\end{array}$ Total } \\
\hline & & Pine & Hardwood & \\
\hline & Number & $-\cdots$ & Standard cords & $3-\cdots$ \\
\hline Virginia & $\begin{array}{l}1 \\
2 \\
3 \\
4 \\
5\end{array}$ & $\begin{array}{r}355,141 \\
421,049 \\
221,107 \\
72,217 \\
10,667 \\
\end{array}$ & $\begin{array}{r}49,750 \\
72,083 \\
53,438 \\
141,363 \\
21,457 \\
\end{array}$ & $\begin{array}{r}404,891 \\
493,132 \\
274,545 \\
213,580 \\
32,124 \\
\end{array}$ \\
\hline Roundwood & & $1,080,181$ & 338,091 & $1,418,272$ \\
\hline Wood residues? & & 97,028 & 3,131 & 100,159 \\
\hline All pulpwoat & & $1,177,209$ & 341,222 & $1,518,431$ \\
\hline North Carolina & $\begin{array}{l}1 \\
2 \\
3 \\
4\end{array}$ & $\begin{array}{l}537,181 \\
274,954 \\
481,657 \\
143,304 \\
\end{array}$ & $\begin{array}{r}68,076 \\
37,594 \\
49,363 \\
170,987 \\
\end{array}$ & $\begin{array}{l}605,257 \\
312,548 \\
531,020 \\
314,291 \\
\end{array}$ \\
\hline Roundwood & & $1,437,096$ & 326,020 & $1,763,116$ \\
\hline Wood residues & & 85,319 & 17,419 & 102,738 \\
\hline All pulpwood & & $1,522,415$ & 343,439 & $1,865.854$ \\
\hline South Carolina & $\begin{array}{l}1 \\
2 \\
3\end{array}$ & $\begin{array}{r}249,524 \\
429,702 \\
705,441 \\
\end{array}$ & $\begin{array}{r}74,765 \\
159,938 \\
35,634 \\
\end{array}$ & $\begin{array}{l}324,289 \\
589,640 \\
741,075 \\
\end{array}$ \\
\hline Roundwood & & $1,384,667$ & 270,337 & $1,655,004$ \\
\hline Wood residues? & & 76,700 & 11,264 & 87,964 \\
\hline All pulpwood & & $1,461,367$ & 281,601 & $1,742,968$ \\
\hline Georgia & $\begin{array}{l}1 \\
2 \\
3 \\
4 \\
5\end{array}$ & $\begin{array}{r}1,486,559 \\
434,721 \\
891,164 \\
560,856 \\
170,048 \\
\end{array}$ & $\begin{array}{r}180,957 \\
24,812 \\
16,503 \\
450 \\
7,306 \\
\end{array}$ & $\begin{array}{r}1,667,516 \\
459,533 \\
907,667 \\
561,306 \\
177,354 \\
\end{array}$ \\
\hline Roundwood & & $3,543,348$ & 230,028 & $3,773,376$ \\
\hline Wood resldues & & 70,375 & 15,259 & 85,634 \\
\hline All pulpwood & & $3,613,723$ & 245,287 & $3,859,010$ \\
\hline Plorida & $\begin{array}{l}1 \\
2 \\
3 \\
4\end{array}$ & $\begin{array}{r}1,202,176 \\
508,717 \\
183,075 \\
27,454 \\
\end{array}$ & $\begin{array}{r}22,467 \\
3,350 \\
140 \\
-- \\
\end{array}$ & $\begin{array}{r}1,224,643 \\
512,067 \\
183,215 \\
27,454 \\
\end{array}$ \\
\hline Roundwood & & $1,921,422$ & 25,957 & $1,947,379$ \\
\hline Wood residues & & 48,933 & 1,181 & 50,114 \\
\hline All pulpwood & & $1,970,355$ & 27,138 & $1,997,493$ \\
\hline
\end{tabular}

\section{Southeastern states}

\begin{tabular}{lrrr}
$\begin{array}{l}\text { Roundwood } \\
\text { Wood residues } 2 /\end{array}$ & $9,366,714$ & $1,190,433$ & $10,557,147$ \\
Total & $\frac{378,355}{48,254}$ & 426,609 \\
\cline { 2 - 4 } & $9,745,069$ & $1,238,687$ & $10,983,756$
\end{tabular}

1) For location of Survey units, see Figure 1 .

2) Residues included in State totals only.
Table 4.--Pulpwood production in Southern Station territory by State and Survey unit, 1957

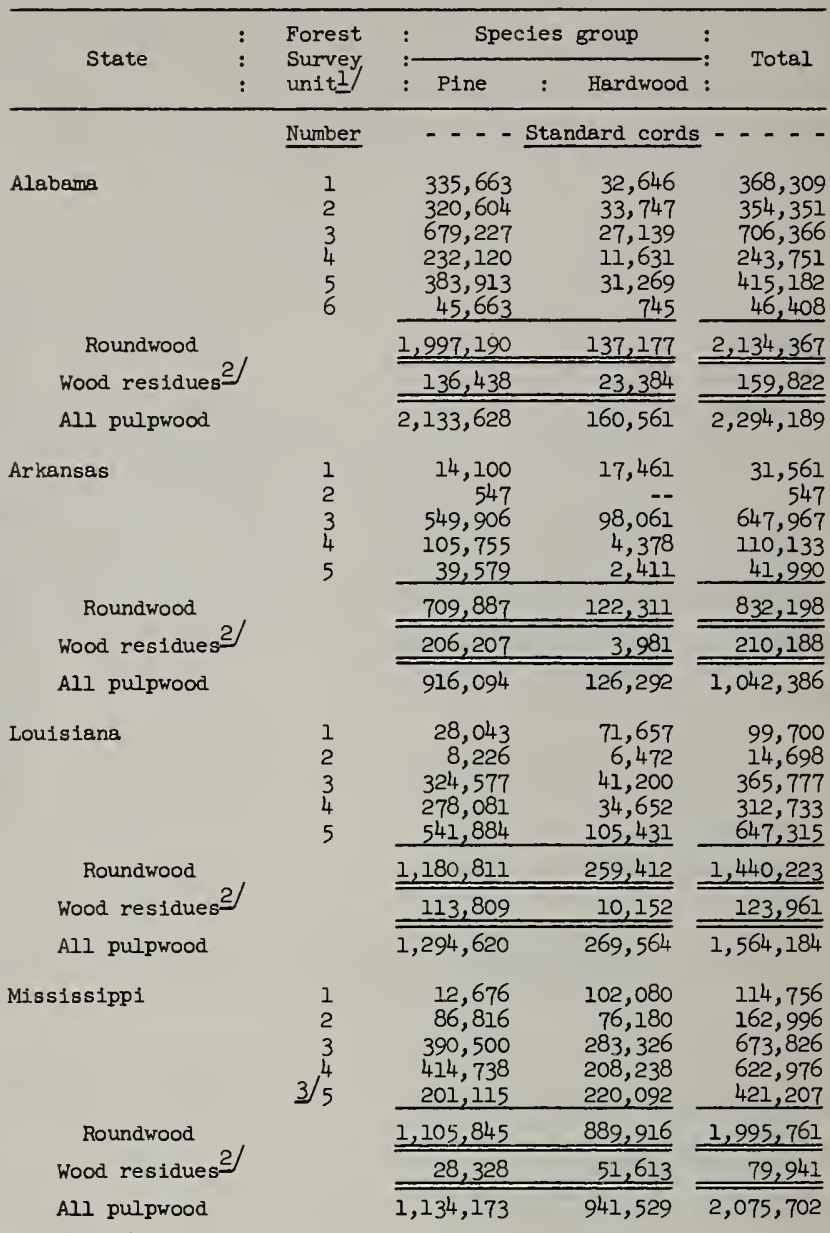

Oklahoma 4 /

Roundwood

Wood residues

All pulpwood

Tennessee

Roundwood

Wood residues $2 /$

All pulpwood

Texas

Roundwood

Wood residues?

All pulpwood

Southern States

Roundwood 2/

Total

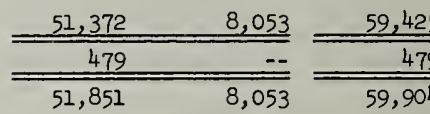

\begin{tabular}{|c|c|}
\hline $\begin{array}{r}9,721 \\
224 \\
137 \\
49,238 \\
176,791 \\
\end{array}$ & $\begin{array}{r}524 \\
6,947 \\
12,652 \\
10,340 \\
67,713 \\
\end{array}$ \\
\hline 236,111 & 98,176 \\
\hline-- & 6,907 \\
\hline 236,111 & $\overline{105,083}$ \\
\hline
\end{tabular}

\begin{tabular}{rrrr}
605,625 & $\begin{array}{r}127,573 \\
44,077\end{array}$ & $\begin{array}{r}733,198 \\
23,720 \\
16,988\end{array}$ & 9,235 \\
$\begin{array}{rr}1,065,690 & 160,528 \\
26,223\end{array}$ & $\frac{1,226,218}{1,297}$ \\
\hline 194,757 & 371 & $\frac{195,128}{1,421,346}$
\end{tabular}

$6,346,906 \quad 1,675,573 \quad 8,022,479$

\begin{tabular}{rrr}
680,018 & 96,408 & 776,426 \\
\hline $7,026,924$ & $1,777,981$ & $8,798,905$
\end{tabular}

1) For location of Survey units, see Figure 1 .

2) Residues included in State totals only.

3/ Survey unit No. 5 has been added in Mississippi.

Present Units No. 3 and No. 5 contain the same area as

Unit No. 3 in the 1956 and earlier reports.

4) All units. 
Table 5.--Round pulpwood production in Alabama, 1957

\begin{tabular}{|c|c|c|c|}
\hline County & Pine & Hardwood & 'Tota]. \\
\hline $\begin{array}{l}\text { Autauga } \\
\text { Baldwin } \\
\text { Barbour } \\
\text { Blbb } \\
\text { Blount }\end{array}$ & $\begin{array}{r}12,754 \\
156,519 \\
46,984 \\
27,972 \\
8,161\end{array}$ & $\begin{array}{r}919 \\
12,949 \\
-- \\
2,892 \\
145\end{array}$ & $\begin{array}{r}13,673 \\
169,468 \\
46,984 \\
30,864 \\
8,306\end{array}$ \\
\hline $\begin{array}{l}\text { Bullock } \\
\text { Butler } \\
\text { Calhoun } \\
\text { Chambers } \\
\text { Cherokee }\end{array}$ & $\begin{array}{l}31,711 \\
61,658 \\
17,300 \\
25,329 \\
18,639\end{array}$ & $\begin{array}{r}134 \\
5,161 \\
920 \\
929 \\
2,635\end{array}$ & $\begin{array}{l}31,845 \\
66,819 \\
18,220 \\
26,258 \\
21,274\end{array}$ \\
\hline $\begin{array}{l}\text { Chilton } \\
\text { Choctaw } \\
\text { Clarke } \\
\text { Clay } \\
\text { Cleburne }\end{array}$ & $\begin{array}{l}25,751 \\
40,840 \\
56,569 \\
40,731 \\
28,259\end{array}$ & $\begin{array}{l}1,212 \\
5,737 \\
7,165 \\
3,858 \\
1,689\end{array}$ & $\begin{array}{l}26,963 \\
46,577 \\
63,734 \\
44,569 \\
29,948\end{array}$ \\
\hline $\begin{array}{l}\text { Coffee } \\
\text { Colbert. } \\
\text { Conecuh } \\
\text { Coosa } \\
\text { Covington }\end{array}$ & $\begin{array}{r}22,549 \\
1,379 \\
38,463 \\
25,780 \\
32,645\end{array}$ & $\begin{array}{r}19 \\
3,880 \\
11,643 \\
326\end{array}$ & $\begin{array}{r}22,549 \\
1,398 \\
42,343 \\
37,423 \\
32,971\end{array}$ \\
\hline $\begin{array}{l}\text { Crenshaw } \\
\text { Cullman } \\
\text { Dale } \\
\text { Dallas } \\
\text { De Kalb }\end{array}$ & $\begin{array}{r}23,151 \\
15,160 \\
18,213 \\
23,984 \\
9,744\end{array}$ & $\begin{array}{r}2,444 \\
141 \\
39 \\
1,802 \\
685\end{array}$ & $\begin{array}{l}25,595 \\
15,301 \\
18,252 \\
25,786 \\
10,429\end{array}$ \\
\hline $\begin{array}{l}\text { Elmore } \\
\text { Escambia } \\
\text { Etowah } \\
\text { Fayette } \\
\text { Franklin }\end{array}$ & $\begin{array}{r}36,614 \\
34,463 \\
16,972 \\
29,042 \\
6,780\end{array}$ & $\begin{array}{r}1,585 \\
3,127 \\
1,025 \\
196 \\
--\end{array}$ & $\begin{array}{r}38,199 \\
37,590 \\
17,997 \\
29,238 \\
6,780\end{array}$ \\
\hline $\begin{array}{l}\text { Geneva } \\
\text { Greene } \\
\text { Hale } \\
\text { Henry } \\
\text { Houston }\end{array}$ & $\begin{array}{r}11,091 \\
24,026 \\
14,184 \\
22,519 \\
6,725\end{array}$ & $\begin{array}{r}781 \\
1,447 \\
17 \\
--\end{array}$ & $\begin{array}{r}11,091 \\
24,807 \\
15,631 \\
22,536 \\
6,725\end{array}$ \\
\hline $\begin{array}{l}\text { Jackson } \\
\text { Jefferson } \\
\text { Lamar } \\
\text { Lauderdale } \\
\text { Lawrence }\end{array}$ & $\begin{array}{r}4,738 \\
26,504 \\
13,747 \\
132 \\
61\end{array}$ & $\begin{array}{r}11 \\
440 \\
82 \\
-- \\
--\end{array}$ & $\begin{array}{r}4,749 \\
26,944 \\
13,829 \\
132 \\
61\end{array}$ \\
\hline $\begin{array}{l}\text { Lee } \\
\text { Limes tone } \\
\text { Lowndes } \\
\text { Macon } \\
\text { Madison }\end{array}$ & $\begin{array}{r}38,902 \\
3,426 \\
55,295 \\
33,648 \\
2,857\end{array}$ & $\begin{array}{r}2,185 \\
-- \\
3,455 \\
53 \\
--\end{array}$ & $\begin{array}{r}41,087 \\
3,426 \\
58,750 \\
33,701 \\
2,857\end{array}$ \\
\hline $\begin{array}{l}\text { Marengo } \\
\text { Marion } \\
\text { Marshall } \\
\text { Mobile } \\
\text { Monroe }\end{array}$ & $\begin{array}{r}35,075 \\
6,480 \\
8,480 \\
49,345 \\
76,218\end{array}$ & $\begin{array}{r}3,542 \\
-- \\
18 \\
11,376 \\
5,492\end{array}$ & $\begin{array}{r}38,617 \\
6,480 \\
8,498 \\
60,721 \\
81,710\end{array}$ \\
\hline $\begin{array}{l}\text { Montgamery } \\
\text { Morgan } \\
\text { Perry } \\
\text { Pickens } \\
\text { Pike }\end{array}$ & $\begin{array}{r}26,423 \\
8,066 \\
33,308 \\
32,875 \\
34,401\end{array}$ & $\begin{array}{r}2,336 \\
12 \\
3,472 \\
768 \\
--\end{array}$ & $\begin{array}{r}28,759 \\
8,078 \\
36,780 \\
33,643 \\
34,401\end{array}$ \\
\hline $\begin{array}{l}\text { Randolph } \\
\text { Russell } \\
\text { St. Clair } \\
\text { Shelby } \\
\text { Sumter }\end{array}$ & $\begin{array}{l}41,896 \\
27,075 \\
27,440 \\
57,626 \\
20,638\end{array}$ & $\begin{array}{r}552 \\
-- \\
954 \\
4,417 \\
2,478\end{array}$ & $\begin{array}{l}42,448 \\
27,075 \\
28,394 \\
62,043 \\
23,116\end{array}$ \\
\hline $\begin{array}{l}\text { Talladega } \\
\text { Tallapoosa } \\
\text { Tuscaloosa } \\
\text { Walker } \\
\text { Washington }\end{array}$ & $\begin{array}{l}27,089 \\
85,685 \\
50,486 \\
32,770 \\
62,691\end{array}$ & $\begin{array}{r}2,852 \\
4,503 \\
1,993 \\
345 \\
4,868\end{array}$ & $\begin{array}{l}29,94+1 \\
90,188 \\
52,479 \\
33,115 \\
67,559\end{array}$ \\
\hline $\begin{array}{l}\text { Wilcox } \\
\text { Winston }\end{array}$ & $\begin{array}{r}52,801 \\
8,371 .\end{array}$ & $\begin{array}{r}5,453 \\
18\end{array}$ & $\begin{array}{r}58,25)^{\prime} \\
8,389\end{array}$ \\
\hline Total. & $1,997,190$ & 137,177 & $2,134,367$ \\
\hline
\end{tabular}

Table 6.--Round pulpwood production in Arkansas, 1.957

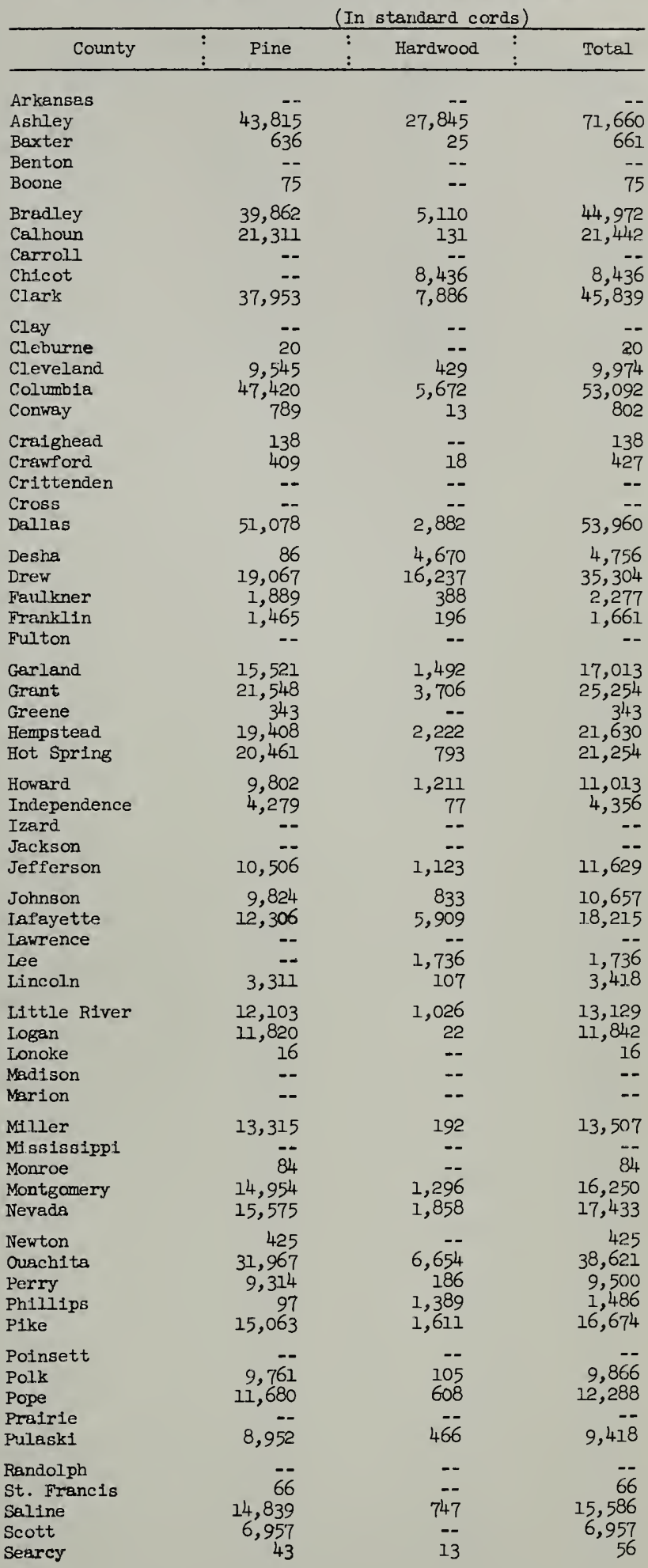



continued

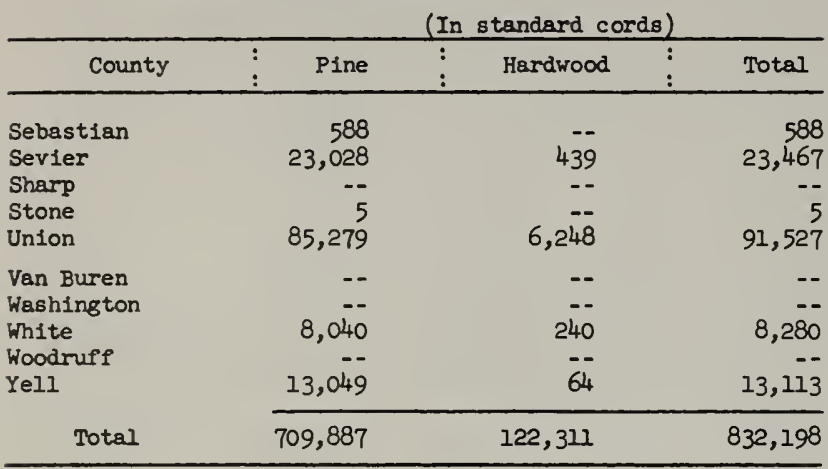

\begin{tabular}{|c|c|c|c|}
\hline County & Pine & Hardwood & Total \\
\hline $\begin{array}{l}\text { Alachua } \\
\text { Baker } \\
\text { Bay } \\
\text { Bradford } \\
\text { Brevard }\end{array}$ & $\begin{array}{l}46,304 \\
86,824 \\
20,602 \\
61,755 \\
18,043\end{array}$ & $\begin{array}{c}595 \\
-- \\
-- \\
53 \\
--\end{array}$ & $\begin{array}{l}46,899 \\
86,824 \\
20,602 \\
61,808 \\
18,043\end{array}$ \\
\hline $\begin{array}{l}\text { Broward } \\
\text { Calhoun } \\
\text { Charlotte } \\
\text { Citrus } \\
\text { Clay }\end{array}$ & $\begin{array}{r}-- \\
35,655 \\
3,678 \\
7,019 \\
71,910\end{array}$ & $\begin{array}{c}-- \\
-- \\
-- \\
1,388\end{array}$ & $\begin{array}{r}-- \\
35,655 \\
3,678 \\
7,019 \\
73,298\end{array}$ \\
\hline $\begin{array}{l}\text { Collier } \\
\text { Columbia } \\
\text { Dade } \\
\text { De Soto } \\
\text { Dixie }\end{array}$ & $\begin{array}{r}8,842 \\
65,172 \\
1,011 \\
7,370 \\
33,595\end{array}$ & $\begin{array}{r}1,130 \\
-- \\
3,300\end{array}$ & $\begin{array}{r}8,842 \\
66,302 \\
1,011 \\
7,370 \\
36,895\end{array}$ \\
\hline $\begin{array}{l}\text { Duval } \\
\text { Escambia } \\
\text { Flagler } \\
\text { Franklin } \\
\text { Gadsden }\end{array}$ & $\begin{array}{l}54,604 \\
51,831 \\
78,486 \\
14,886 \\
28,884\end{array}$ & $\begin{array}{r}1,100 \\
12 \\
-- \\
-- \\
2,040\end{array}$ & $\begin{array}{l}55,704 \\
51,843 \\
78,486 \\
14,886 \\
30,924\end{array}$ \\
\hline $\begin{array}{l}\text { Gilchrist } \\
\text { Glades } \\
\text { Gulf } \\
\text { Hamilton } \\
\text { Hardee }\end{array}$ & $\begin{array}{r}12,912 \\
2,996 \\
27,448 \\
72,488 \\
7,739\end{array}$ & $\begin{array}{r}-- \\
-- \\
1,134 \\
--\end{array}$ & $\begin{array}{r}12,912 \\
2,996 \\
27,448 \\
73,622 \\
7,739\end{array}$ \\
\hline $\begin{array}{l}\text { Hendry } \\
\text { Hernando } \\
\text { Highlands } \\
\text { Hillsborough } \\
\text { Holmes }\end{array}$ & $\begin{array}{r}6,373 \\
8,221 \\
3,400 \\
25,877 \\
28,111\end{array}$ & $\begin{array}{r}-- \\
105 \\
-- \\
-- \\
--\end{array}$ & $\begin{array}{r}6,373 \\
8,326 \\
3,400 \\
25,877 \\
28,111\end{array}$ \\
\hline $\begin{array}{l}\text { Indian River } \\
\text { Jackson } \\
\text { Jefferson } \\
\text { Lafayette } \\
\text { Lake }\end{array}$ & $\begin{array}{r}114 \\
46,550 \\
22,587 \\
26,958 \\
8,282\end{array}$ & $\begin{array}{r}-- \\
-- \\
750 \\
597 \\
--\end{array}$ & $\begin{array}{r}114 \\
46,550 \\
23,337 \\
27,555 \\
8,282\end{array}$ \\
\hline $\begin{array}{l}\text { Lee } \\
\text { Leon } \\
\text { Levy } \\
\text { Liberty } \\
\text { Madison }\end{array}$ & $\begin{array}{r}3,830 \\
25,362 \\
55,446 \\
24,288 \\
41,346\end{array}$ & 2,000 & $\begin{array}{r}3,830 \\
25,497 \\
55,446 \\
24,288 \\
43,346\end{array}$ \\
\hline $\begin{array}{l}\text { Manatee } \\
\text { Marion } \\
\text { Martin } \\
\text { Monroe } \\
\text { Nassau }\end{array}$ & $\begin{array}{r}1,260 \\
81,939 \\
689 \\
-- \\
77,222\end{array}$ & $\begin{array}{r}1,054 \\
-- \\
1,579\end{array}$ & $\begin{array}{r}1,260 \\
82,993 \\
689 \\
=- \\
78,801\end{array}$ \\
\hline $\begin{array}{l}\text { Okaloosa } \\
\text { Okeechobee } \\
\text { Orange } \\
\text { Osceola } \\
\text { Palm Beach }\end{array}$ & $\begin{array}{r}21,049 \\
3,652 \\
13,062 \\
16,652 \\
35\end{array}$ & $\begin{array}{l}-- \\
-- \\
-- \\
--\end{array}$ & $\begin{array}{r}21,049 \\
3,652 \\
13,062 \\
16,652 \\
35\end{array}$ \\
\hline $\begin{array}{l}\text { Pasco } \\
\text { Pinellas } \\
\text { Polk } \\
\text { Putnam } \\
\text { St. Johns }\end{array}$ & $\begin{array}{r}10,306 \\
105 \\
20,610 \\
63,369 \\
78,452\end{array}$ & $\begin{array}{r}35 \\
-- \\
5,000 \\
2,937\end{array}$ & $\begin{array}{r}10,341 \\
105 \\
20,610 \\
68,369 \\
81,389\end{array}$ \\
\hline $\begin{array}{l}\text { St. Lucie } \\
\text { Santa Rosa } \\
\text { Sarasota } \\
\text { Seminole } \\
\text { Sumter }\end{array}$ & $\begin{array}{r}704 \\
82,669 \\
8,678 \\
10,245 \\
11,736\end{array}$ & $\begin{array}{l}-- \\
5 \\
-- \\
-- \\
--\end{array}$ & $\begin{array}{r}704 \\
82,674 \\
8,678 \\
10,245 \\
11,736\end{array}$ \\
\hline $\begin{array}{l}\text { Suwannee } \\
\text { Taylor } \\
\text { Union } \\
\text { Volusia } \\
\text { Wakulla }\end{array}$ & $\begin{array}{l}36,250 \\
52,468 \\
42,610 \\
62,066 \\
15,809\end{array}$ & $\begin{array}{r}100 \\
500 \\
-- \\
-- \\
400\end{array}$ & $\begin{array}{l}36,350 \\
52,968 \\
42,610 \\
62,066 \\
16,209\end{array}$ \\
\hline $\begin{array}{l}\text { Walton } \\
\text { Wash1ngton }\end{array}$ & $\begin{array}{l}33,122 \\
29,864\end{array}$ & $\begin{array}{c}8 \\
--\end{array}$ & $\begin{array}{l}33,130 \\
29,864\end{array}$ \\
\hline Total & $1,921,422$ & 25,957 & $1,947,379$ \\
\hline
\end{tabular}


Table 8.--Round pulpwood production in Ceorg1a, 1957

\begin{tabular}{|c|c|c|c|}
\hline County & Pine & Hardwood & Total \\
\hline $\begin{array}{l}\text { Appling } \\
\text { Atkinson } \\
\text { Bacon } \\
\text { Baker } \\
\text { Baldwin }\end{array}$ & $\begin{array}{r}75,070 \\
52,780 \\
33,694 \\
8,030 \\
18,913\end{array}$ & $\begin{array}{r}2,426 \\
369 \\
1,285 \\
-- \\
684\end{array}$ & $\begin{array}{r}77,496 \\
53,149 \\
34,979 \\
8,030 \\
19,597\end{array}$ \\
\hline $\begin{array}{l}\text { Banks } \\
\text { Barrow } \\
\text { Bartow } \\
\text { Ben Hill } \\
\text { Berrien }\end{array}$ & $\begin{array}{r}4,386 \\
4,031 \\
18,413 \\
12,184 \\
41,238\end{array}$ & $\begin{array}{r}-- \\
2,348 \\
1,781 \\
1,851\end{array}$ & $\begin{array}{r}4,386 \\
4,031 \\
20,761 \\
13,965 \\
43,089\end{array}$ \\
\hline $\begin{array}{l}\text { B1bb } \\
\text { Bleckley } \\
\text { Brantley } \\
\text { Brooks } \\
\text { Bryan }\end{array}$ & $\begin{array}{r}7,595 \\
4,038 \\
69,969 \\
16,841 \\
51,301\end{array}$ & $\begin{array}{r}1,626 \\
1,221 \\
5,235 \\
200 \\
15,016\end{array}$ & $\begin{array}{r}9,221 \\
5,259 \\
75,204 \\
17,041 \\
66,317\end{array}$ \\
\hline $\begin{array}{l}\text { Bulloch } \\
\text { Burke } \\
\text { Butts } \\
\text { Calhoun } \\
\text { Camden }\end{array}$ & $\begin{array}{r}24,949 \\
15,020 \\
27,116 \\
4,837 \\
76,234\end{array}$ & $\begin{array}{r}2,800 \\
367 \\
70 \\
=- \\
25,320\end{array}$ & $\begin{array}{r}27,749 \\
15,387 \\
27,186 \\
4,837 \\
101,554\end{array}$ \\
\hline $\begin{array}{l}\text { Candler } \\
\text { Carroll } \\
\text { Catoosa } \\
\text { Charlton } \\
\text { Chatham }\end{array}$ & $\begin{array}{r}6,256 \\
33,095 \\
2,388 \\
77,123 \\
8,211\end{array}$ & $\begin{array}{r}800 \\
30 \\
3 \\
4,517 \\
4,074\end{array}$ & $\begin{array}{r}7,056 \\
33,125 \\
2,391 \\
81,634 \\
12,285\end{array}$ \\
\hline $\begin{array}{l}\text { Chattahoochee } \\
\text { Chattooga } \\
\text { Cherokee } \\
\text { Clarke } \\
\text { Clay }\end{array}$ & $\begin{array}{r}12,923 \\
8,885 \\
14,602 \\
2,366 \\
9,398\end{array}$ & $\begin{array}{r}622 \\
268 \\
-- \\
--\end{array}$ & $\begin{array}{r}12,923 \\
9,507 \\
14,870 \\
2,366 \\
9,398\end{array}$ \\
\hline $\begin{array}{l}\text { Clayton } \\
\text { Clinch } \\
\text { Cobb } \\
\text { Coffee } \\
\text { Colquitt }\end{array}$ & $\begin{array}{r}8,576 \\
99,905 \\
16,116 \\
59,481 \\
37,078\end{array}$ & $\begin{array}{r}295 \\
14 \\
1,921 \\
909\end{array}$ & $\begin{array}{r}8,576 \\
100,200 \\
16,130 \\
61,402 \\
37,987\end{array}$ \\
\hline $\begin{array}{l}\text { Columb1a } \\
\text { Cook } \\
\text { Coweta } \\
\text { Crawford } \\
\text { Crisp }\end{array}$ & $\begin{array}{r}22,548 \\
21,643 \\
40,246 \\
21,293 \\
8,010\end{array}$ & $\begin{array}{r}821 \\
35 \\
-- \\
970 \\
150\end{array}$ & $\begin{array}{r}23,369 \\
21,678 \\
40,246 \\
22,263 \\
8,160\end{array}$ \\
\hline $\begin{array}{l}\text { Dade } \\
\text { Dawson } \\
\text { Decatur } \\
\text { De Kalb } \\
\text { Dodge }\end{array}$ & $\begin{array}{r}1,115 \\
2,508 \\
54,590 \\
6,232 \\
41,436\end{array}$ & $\begin{array}{r}101 \\
=- \\
6,159 \\
1,537\end{array}$ & $\begin{array}{r}1,216 \\
2,508 \\
60,749 \\
6,232 \\
42,973\end{array}$ \\
\hline $\begin{array}{l}\text { Dooly } \\
\text { Dougherty } \\
\text { Douglas } \\
\text { Early } \\
\text { Echols }\end{array}$ & $\begin{array}{r}13,522 \\
14,259 \\
27,660 \\
7,816 \\
42,419\end{array}$ & $\begin{array}{r}62 \\
-- \\
12 \\
464 \\
11\end{array}$ & $\begin{array}{r}13,584 \\
14,259 \\
27,672 \\
8,280 \\
42,430\end{array}$ \\
\hline $\begin{array}{l}\text { Efflngham } \\
\text { Elbert } \\
\text { Emanuel } \\
\text { Evans } \\
\text { Fannin }\end{array}$ & $\begin{array}{r}40,318 \\
24,015 \\
40,361 \\
6,612 \\
4,615\end{array}$ & $\begin{array}{r}9,739 \\
-- \\
1,272 \\
50 \\
1,260\end{array}$ & $\begin{array}{r}50,057 \\
24,015 \\
41,633 \\
6,662 \\
5,875\end{array}$ \\
\hline $\begin{array}{l}\text { Fayette } \\
\text { Floyd } \\
\text { Forsyth } \\
\text { Franklin } \\
\text { Fulton }\end{array}$ & $\begin{array}{r}7,304 \\
23,163 \\
2,126 \\
13,717 \\
16,576\end{array}$ & $\begin{array}{r}1,740 \\
-- \\
- \\
35\end{array}$ & $\begin{array}{r}7,304 \\
24,903 \\
2,126 \\
13,717 \\
16,611\end{array}$ \\
\hline $\begin{array}{l}\text { Gilmer } \\
\text { Glascock } \\
\text { Glynn } \\
\text { Gordon } \\
\text { Grady }\end{array}$ & $\begin{array}{r}6,912 \\
2,550 \\
39,329 \\
20,520 \\
24,969\end{array}$ & $\begin{array}{r}337 \\
\ldots \\
24,443 \\
327 \\
1,773\end{array}$ & $\begin{array}{r}7,249 \\
2,550 \\
63,772 \\
20,847 \\
26,742\end{array}$ \\
\hline
\end{tabular}

Table 8.--Round pulpwood production in Georg1a, 1957 continued

\begin{tabular}{|c|c|c|c|c|}
\hline County & Pine & : $\quad$ Bardwood & : & Total \\
\hline $\begin{array}{l}\text { Greene } \\
\text { Grimett } \\
\text { Habersham } \\
\text { Hall } \\
\text { Hancock }\end{array}$ & $\begin{array}{r}56,493 \\
27,579 \\
8,806 \\
15,372 \\
37,148\end{array}$ & $\begin{array}{r}1,000 \\
\ldots \\
\ldots \\
1,068\end{array}$ & & $\begin{array}{r}57,493 \\
27,579 \\
8,806 \\
15,372 \\
38,216\end{array}$ \\
\hline $\begin{array}{l}\text { Haralson } \\
\text { Harris } \\
\text { Hart } \\
\text { Heard } \\
\text { Henry }\end{array}$ & $\begin{array}{r}10,699 \\
41,221 \\
7,310 \\
7,958 \\
29,373\end{array}$ & $\begin{array}{r}176 \\
100 \\
-- \\
-- \\
50\end{array}$ & & $\begin{array}{r}10,875 \\
41,321 \\
7,310 \\
7,958 \\
29,423\end{array}$ \\
\hline $\begin{array}{l}\text { Houston } \\
\text { Irwin } \\
\text { Jackson } \\
\text { Jasper } \\
\text { Jeff Davis }\end{array}$ & $\begin{array}{l}19,259 \\
15,200 \\
13,830 \\
23,231 \\
34,714\end{array}$ & $\begin{array}{r}466 \\
2,738 \\
16 \\
835 \\
505\end{array}$ & & $\begin{array}{l}19,725 \\
17,938 \\
13,846 \\
24,066 \\
35,219\end{array}$ \\
\hline $\begin{array}{l}\text { Jefferson } \\
\text { Jenkins } \\
\text { Johnson } \\
\text { Jones } \\
\text { Iamar }\end{array}$ & $\begin{array}{r}23,914 \\
8,569 \\
7,844 \\
45,355 \\
11,179\end{array}$ & $\begin{array}{r}581 \\
550 \\
405 \\
817 \\
49\end{array}$ & & $\begin{array}{r}24,495 \\
9,119 \\
8,249 \\
46,172 \\
11,228\end{array}$ \\
\hline $\begin{array}{l}\text { Lanier } \\
\text { Laurens } \\
\text { Lee } \\
\text { Liberty } \\
\text { Lincoln }\end{array}$ & $\begin{array}{r}10,157 \\
22,972 \\
5,658 \\
24,043 \\
10,006\end{array}$ & $\begin{array}{r}250 \\
717 \\
-- \\
18,693 \\
--\end{array}$ & & $\begin{array}{r}10,407 \\
23,689 \\
5,658 \\
42,736 \\
10,006\end{array}$ \\
\hline $\begin{array}{l}\text { Long } \\
\text { Lowndes } \\
\text { Lumpin } \\
\text { McDuffle } \\
\text { McIntosh }\end{array}$ & $\begin{array}{r}36,832 \\
22,284 \\
2,302 \\
12,662 \\
26,075\end{array}$ & $\begin{array}{r}3,104 \\
799 \\
- \\
250 \\
25,575\end{array}$ & & $\begin{array}{r}39,936 \\
23,083 \\
2,302 \\
12,912 \\
51,650\end{array}$ \\
\hline $\begin{array}{l}\text { Macon } \\
\text { Madison } \\
\text { Marion } \\
\text { Meriwether } \\
\text { Miller }\end{array}$ & $\begin{array}{r}11,718 \\
8,760 \\
9,168 \\
40,652 \\
12,303\end{array}$ & $\begin{array}{r}144 \\
-- \\
-- \\
-- \\
--\end{array}$ & & $\begin{array}{r}11,862 \\
8,760 \\
9,168 \\
40,652 \\
12,303\end{array}$ \\
\hline $\begin{array}{l}\text { Mitchell } \\
\text { Monroe } \\
\text { Montgomery } \\
\text { Morgan } \\
\text { Murray }\end{array}$ & $\begin{array}{r}32,667 \\
43,219 \\
12,505 \\
15,435 \\
5,812\end{array}$ & $\begin{array}{r}2,267 \\
773 \\
2,681 \\
- \\
37\end{array}$ & & $\begin{array}{r}34,934 \\
43,992 \\
15,186 \\
15,435 \\
5,849\end{array}$ \\
\hline $\begin{array}{l}\text { Muscogee } \\
\text { Newton } \\
\text { Oconee } \\
\text { Oglethorpe } \\
\text { Paulding }\end{array}$ & $\begin{array}{r}8,981 \\
36,040 \\
12,197 \\
20,549 \\
24,652\end{array}$ & $\begin{array}{l}-. \\
20 \\
-- \\
--\end{array}$ & & $\begin{array}{r}8,981 \\
36,060 \\
12,197 \\
20,549 \\
24,652\end{array}$ \\
\hline $\begin{array}{l}\text { Peach } \\
\text { P1ckens } \\
\text { Plerce } \\
\text { P1ke } \\
\text { Polk }\end{array}$ & $\begin{array}{r}8,094 \\
8,800 \\
104,315 \\
10,108 \\
15,081\end{array}$ & $\begin{array}{r}426 \\
2,646 \\
-- \\
97\end{array}$ & & $\begin{array}{r}8,520 \\
8,800 \\
106,961 \\
10,108 \\
15,178\end{array}$ \\
\hline $\begin{array}{l}\text { Pulask1 } \\
\text { Putnam } \\
\text { Quitman } \\
\text { Rabun } \\
\text { Randolph }\end{array}$ & $\begin{array}{r}4,573 \\
33,170 \\
8,933 \\
2,449 \\
11,301\end{array}$ & $\begin{array}{r}115 \\
931 \\
-- \\
157 \\
--\end{array}$ & & $\begin{array}{r}4,688 \\
34,101 \\
8,933 \\
2,606 \\
11,301\end{array}$ \\
\hline $\begin{array}{l}\text { Richmond } \\
\text { Rockdale } \\
\text { Schley } \\
\text { Screven } \\
\text { Seminole }\end{array}$ & $\begin{array}{r}4,403 \\
5,805 \\
12,103 \\
18,758 \\
18,801\end{array}$ & $\begin{array}{r}198 \\
-- \\
-- \\
3,217 \\
1,313\end{array}$ & & $\begin{array}{r}4,601 \\
5,805 \\
12,103 \\
21,975 \\
20,114\end{array}$ \\
\hline $\begin{array}{l}\text { Spalding } \\
\text { Stephens } \\
\text { Stewart } \\
\text { Sumter } \\
\text { Trlbot }\end{array}$ & $\begin{array}{l}12,673 \\
11,849 \\
26,886 \\
19,949 \\
33,865\end{array}$ & $\begin{array}{r}-- \\
50 \\
300 \\
300\end{array}$ & & $\begin{array}{l}12,673 \\
11,849 \\
26,936 \\
20,249 \\
34,165\end{array}$ \\
\hline
\end{tabular}


Table 8.--Round pulpwood product1on in Georg1a, 1957 continued

\begin{tabular}{|c|c|c|c|}
\hline County & Pine & : Hardwood & Total \\
\hline $\begin{array}{l}\text { Taliaferro } \\
\text { Tattnall } \\
\text { Taylor } \\
\text { Telfair } \\
\text { Terrell }\end{array}$ & $\begin{array}{r}7,431 \\
45,982 \\
12,042 \\
42,545 \\
4,492\end{array}$ & $\begin{array}{r}16 \\
3,929 \\
100 \\
1,574 \\
--\end{array}$ & $\begin{array}{r}7,447 \\
49,911 \\
12,142 \\
44,119 \\
4,492\end{array}$ \\
\hline $\begin{array}{l}\text { Thamas } \\
\text { Tift } \\
\text { Toombs } \\
\text { Towns } \\
\text { Treutlen }\end{array}$ & $\begin{array}{r}29,360 \\
8,360 \\
23,769 \\
-- \\
15,015\end{array}$ & $\begin{array}{r}2,526 \\
818 \\
3,429 \\
-- \\
68\end{array}$ & $\begin{array}{r}31,886 \\
9,178 \\
27,198 \\
15,083\end{array}$ \\
\hline $\begin{array}{l}\text { Troup } \\
\text { Turner } \\
\text { Twiggs } \\
\text { Unton } \\
\text { Upson }\end{array}$ & $\begin{array}{r}58,002 \\
6,481 \\
18,928 \\
2,867 \\
30,451\end{array}$ & $\begin{array}{r}- \\
50 \\
560 \\
-\overline{-} \\
108\end{array}$ & $\begin{array}{r}58,002 \\
6,531 \\
19,488 \\
2,867 \\
30,559\end{array}$ \\
\hline $\begin{array}{l}\text { Walker } \\
\text { Walton } \\
\text { Ware } \\
\text { Warren } \\
\text { Washington }\end{array}$ & $\begin{array}{r}6,120 \\
7,878 \\
88,022 \\
15,896 \\
25,727\end{array}$ & $\begin{array}{r}106 \\
-- \\
646 \\
50 \\
150\end{array}$ & $\begin{array}{r}6,226 \\
7,878 \\
88,668 \\
15,946 \\
25,877\end{array}$ \\
\hline $\begin{array}{l}\text { Wayne } \\
\text { Webster } \\
\text { Wheeler } \\
\text { White } \\
\text { Whitfield }\end{array}$ & $\begin{array}{r}96,257 \\
8,535 \\
32,894 \\
2,840 \\
15,082\end{array}$ & $\begin{array}{r}10,387 \\
25 \\
1,727 \\
-- \\
--\end{array}$ & $\begin{array}{r}106,644 \\
8,560 \\
34,621 \\
2,840 \\
15,082\end{array}$ \\
\hline $\begin{array}{l}\text { Wilcox } \\
\text { Wilkes } \\
\text { Wilkinson } \\
\text { Worth }\end{array}$ & $\begin{array}{l}16,206 \\
32,758 \\
26,382 \\
16,981\end{array}$ & $\begin{array}{l}442 \\
869 \\
463 \\
225\end{array}$ & $\begin{array}{l}16,648 \\
33,627 \\
26,845 \\
17,206\end{array}$ \\
\hline Total & $3,543,348$ & 230,028 & $3,773,376$ \\
\hline
\end{tabular}


Table 10.--Round pulpwood production in Mississ1pp1, 1957

\begin{tabular}{|c|c|c|c|}
\hline County & Pine & Hardwood & Total \\
\hline $\begin{array}{l}\text { Adams } \\
\text { Alcorn } \\
\text { Amfte } \\
\text { Attala } \\
\text { Benton }\end{array}$ & $\begin{array}{r}5,128 \\
4,083 \\
18,539 \\
14,679 \\
1,677\end{array}$ & $\begin{array}{r}17,203 \\
388 \\
15,172 \\
21,902 \\
383\end{array}$ & $\begin{array}{r}22,331 \\
4,471 \\
33,711 \\
36,581 \\
2,060\end{array}$ \\
\hline $\begin{array}{l}\text { Bolivar } \\
\text { Calhoun } \\
\text { Carroll } \\
\text { Chickasaw } \\
\text { Choctaw }\end{array}$ & $\begin{array}{r}-- \\
229 \\
2,227 \\
5,612 \\
10,908\end{array}$ & $\begin{array}{r}6,429 \\
1,104 \\
11,091 \\
2,060 \\
6,334\end{array}$ & $\begin{array}{r}6,429 \\
1,333 \\
13,318 \\
7,672 \\
17,242\end{array}$ \\
\hline $\begin{array}{l}\text { Claiborne } \\
\text { Clarke } \\
\text { Clay } \\
\text { Coahoma } \\
\text { Copiah }\end{array}$ & $\begin{array}{r}6,240 \\
46,332 \\
381 \\
-- \\
31,214\end{array}$ & $\begin{array}{r}24,702 \\
33,724 \\
98 \\
4,698 \\
36,225\end{array}$ & $\begin{array}{r}30,942 \\
80,056 \\
479 \\
4,698 \\
67,439\end{array}$ \\
\hline $\begin{array}{l}\text { Covington } \\
\text { De Soto } \\
\text { Forrest } \\
\text { Franklin } \\
\text { George }\end{array}$ & $\begin{array}{r}21,019 \\
=- \\
23,634 \\
34,094 \\
16,013\end{array}$ & $\begin{array}{r}10,492 \\
-- \\
17,146 \\
16,577 \\
1,901\end{array}$ & $\begin{array}{r}31,511 \\
40,780 \\
50,671 \\
17,914\end{array}$ \\
\hline $\begin{array}{l}\text { Greene } \\
\text { Grenada } \\
\text { Hancock } \\
\text { Harrison } \\
\text { Hinds }\end{array}$ & $\begin{array}{r}24,615 \\
1,214 \\
44,741 \\
30,940 \\
12,325\end{array}$ & $\begin{array}{r}15,545 \\
4,362 \\
1,010 \\
1,653 \\
24,224\end{array}$ & $\begin{array}{r}40,160 \\
5,576 \\
45,751 \\
32,593 \\
36,549\end{array}$ \\
\hline $\begin{array}{l}\text { Holmes } \\
\text { Humphreys } \\
\text { Issaquena } \\
\text { Itawamba } \\
\text { Jackson }\end{array}$ & $\begin{array}{r}10,821 \\
-- \\
\overline{86} \\
40,534\end{array}$ & $\begin{array}{r}23,555 \\
6,854 \\
11,263 \\
=- \\
268\end{array}$ & $\begin{array}{r}34,376 \\
6,854 \\
11,263 \\
86 \\
40,802\end{array}$ \\
\hline $\begin{array}{l}\text { Jasper } \\
\text { Jefferson } \\
\text { Jefferson Davis } \\
\text { Jones } \\
\text { Kemper }\end{array}$ & $\begin{array}{l}43,982 \\
14,520 \\
14,687 \\
17,942 \\
24,020\end{array}$ & $\begin{array}{r}45,166 \\
18,776 \\
5,187 \\
35,371 \\
6,091\end{array}$ & $\begin{array}{l}89,148 \\
33,296 \\
19,874 \\
53,313 \\
30,111\end{array}$ \\
\hline $\begin{array}{l}\text { Lafayette } \\
\text { Lamar } \\
\text { Lauderdale } \\
\text { Lawrence } \\
\text { Leake }\end{array}$ & $\begin{array}{r}3,736 \\
12,923 \\
48,512 \\
30,987 \\
25,474\end{array}$ & $\begin{array}{r}5,679 \\
7,159 \\
17,177 \\
21,532 \\
31,372\end{array}$ & $\begin{array}{r}9,415 \\
20,082 \\
65,689 \\
52,519 \\
56,846\end{array}$ \\
\hline $\begin{array}{l}\text { Lee } \\
\text { Leflore } \\
\text { Lincoln } \\
\text { Lowndes } \\
\text { Madison }\end{array}$ & $\begin{array}{r}1,767 \\
161 \\
29,987 \\
3,774 \\
6,431\end{array}$ & $\begin{array}{r}106 \\
1,644 \\
22,299 \\
2 \\
11,283\end{array}$ & $\begin{array}{r}1,873 \\
1,805 \\
52,286 \\
3,776 \\
17,714\end{array}$ \\
\hline $\begin{array}{l}\text { Marion } \\
\text { Marshall } \\
\text { Monroe } \\
\text { Montgomery } \\
\text { Neshoba }\end{array}$ & $\begin{array}{r}25,356 \\
2,304 \\
3,447 \\
2,688 \\
20,696\end{array}$ & $\begin{array}{r}26,901 \\
2,629 \\
292 \\
8,187 \\
20,176\end{array}$ & $\begin{array}{r}52,257 \\
4,933 \\
3,739 \\
10,875 \\
40,872\end{array}$ \\
\hline $\begin{array}{l}\text { Newton } \\
\text { Noxubee } \\
\text { Oktibbeha } \\
\text { Panola } \\
\text { Pearl River }\end{array}$ & $\begin{array}{r}35,021 \\
11,427 \\
10,799 \\
14 \\
24,899\end{array}$ & $\begin{array}{r}18,303 \\
706 \\
7,238 \\
4,833 \\
15,850\end{array}$ & $\begin{array}{r}53,324 \\
12,133 \\
18,037 \\
4,847 \\
40,749\end{array}$ \\
\hline $\begin{array}{l}\text { Perry } \\
\text { Pike } \\
\text { Pontotoc } \\
\text { Prentiss } \\
\text { Quitman }\end{array}$ & $\begin{array}{r}21,909 \\
33,830 \\
7,001 \\
5,365 \\
--\end{array}$ & $\begin{array}{r}14,326 \\
19,156 \\
2,132 \\
681 \\
3,188\end{array}$ & $\begin{array}{r}36,235 \\
52,986 \\
9,133 \\
6,046 \\
3,188\end{array}$ \\
\hline $\begin{array}{l}\text { Rankin } \\
\text { Scott } \\
\text { Sharkey } \\
\text { Simpson } \\
\text { Smith }\end{array}$ & $\begin{array}{r}27,373 \\
27,829 \\
-- \\
21,167 \\
26,217\end{array}$ & $\begin{array}{r}20,018 \\
12,970 \\
5,069 \\
24,758 \\
21,139\end{array}$ & $\begin{array}{r}47,391 \\
40,799 \\
5,069 \\
45,925 \\
47,356\end{array}$ \\
\hline
\end{tabular}

Table 10.--Round pulpwood production in Mississippi, 1957 (continued)

\begin{tabular}{|c|c|c|c|}
\hline County & Pine & Hardwood & Total \\
\hline $\begin{array}{l}\text { Stone } \\
\text { Sunflower } \\
\text { Tailahatchie } \\
\text { Tate } \\
\text { Tippah }\end{array}$ & $\begin{array}{r}17,330 \\
-- \\
297 \\
60 \\
2,671\end{array}$ & $\begin{array}{r}9,070 \\
18 \\
4,378 \\
1,577 \\
630\end{array}$ & $\begin{array}{r}26,400 \\
18 \\
4,675 \\
1,637 \\
3,301\end{array}$ \\
\hline $\begin{array}{l}\text { Tishomingo } \\
\text { Tunica } \\
\text { Union } \\
\text { Walthall } \\
\text { Warren }\end{array}$ & $\begin{array}{r}6,840 \\
2 . \\
2,544 \\
16,361 \\
948\end{array}$ & $\begin{array}{r}174 \\
235 \\
788 \\
5,828 \\
22,854\end{array}$ & $\begin{array}{r}7,014 \\
235 \\
3,332 \\
22,189 \\
23,802\end{array}$ \\
\hline $\begin{array}{l}\text { Washington } \\
\text { Wayne } \\
\text { Webster } \\
\text { Wilkinson } \\
\text { Winston }\end{array}$ & $\begin{array}{r}30,848 \\
4,367 \\
8,807 \\
17,771\end{array}$ & $\begin{array}{r}4,248 \\
18,999 \\
5,193 \\
14,475 \\
9,824\end{array}$ & $\begin{array}{r}4,248 \\
49,847 \\
9,560 \\
23,282 \\
27,595\end{array}$ \\
\hline $\begin{array}{l}\text { Yalobusha } \\
\text { Yazoo }\end{array}$ & $\begin{array}{r}3,022 \\
449\end{array}$ & $\begin{array}{r}10,219 \\
7,647\end{array}$ & $\begin{array}{r}13,241 \\
8,096\end{array}$ \\
\hline Total & $1,105,845$ & 889,916 & $1,995,761$ \\
\hline
\end{tabular}


Table 11.--Round pulpwood production in North Caroling, 1957

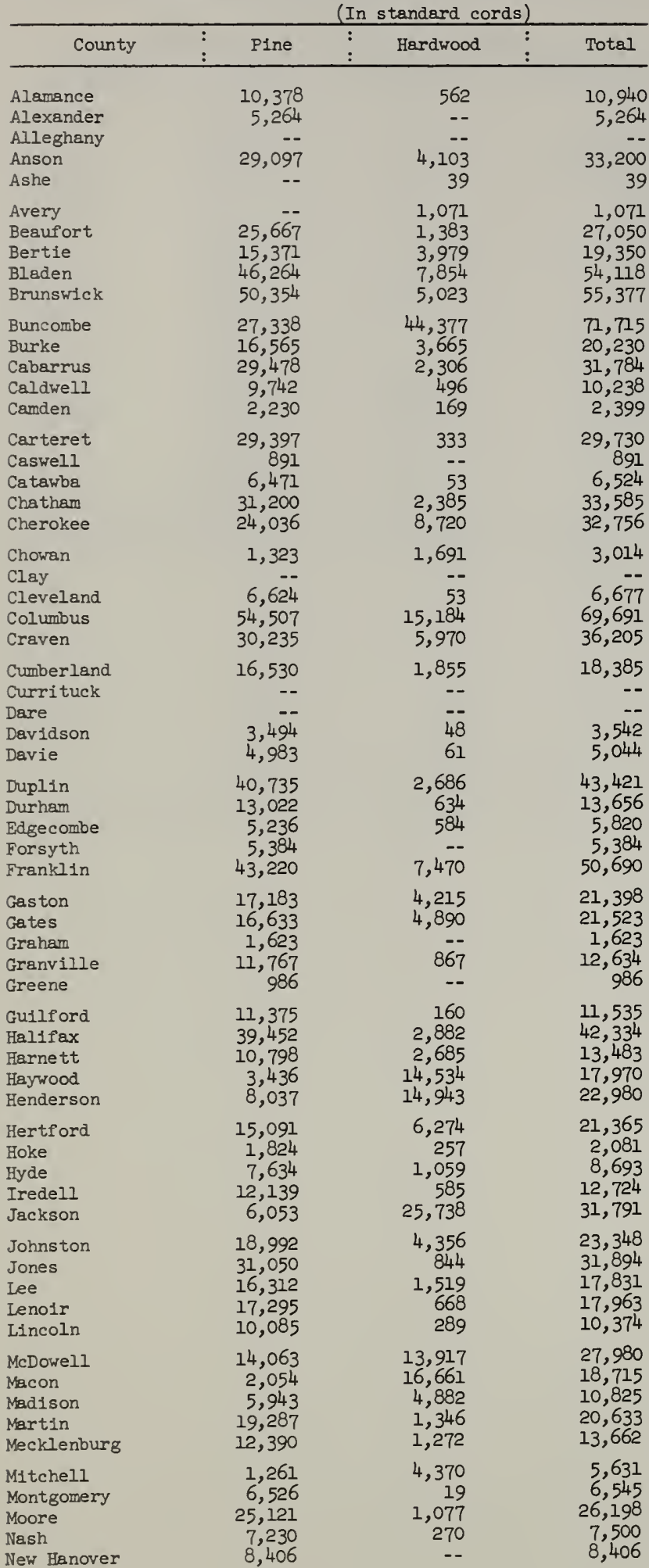

Table 11.--Round pulpwood production in North Carolina, 1957 (continued)

\begin{tabular}{|c|c|c|c|}
\hline County & PIne & Hardwood & Total \\
\hline $\begin{array}{l}\text { Northampton } \\
\text { Onslow } \\
\text { Orange } \\
\text { Pamlico } \\
\text { Pasquotank }\end{array}$ & $\begin{array}{r}12,994 \\
67,076 \\
16,865 \\
8,694 \\
124\end{array}$ & $\begin{array}{r}3,627 \\
674 \\
142 \\
508 \\
68\end{array}$ & $\begin{array}{r}16,621 \\
67,750 \\
17,007 \\
9,202 \\
192\end{array}$ \\
\hline $\begin{array}{l}\text { Pender } \\
\text { Perquimans } \\
\text { Person } \\
\text { P1tt } \\
\text { Polk }\end{array}$ & $\begin{array}{r}42,276 \\
1,057 \\
17,501 \\
5,995 \\
5,031\end{array}$ & $\begin{array}{r}8,545 \\
69 \\
\overline{83} \\
11,758\end{array}$ & $\begin{array}{r}50,821 \\
1,126 \\
17,501 \\
6,078 \\
16,789\end{array}$ \\
\hline $\begin{array}{l}\text { Randolph } \\
\text { Richmond } \\
\text { Robeson } \\
\text { Rockingham } \\
\text { Rowan }\end{array}$ & $\begin{array}{r}6,831 \\
19,992 \\
27,487 \\
6,176 \\
3,794\end{array}$ & $\begin{array}{r}-- \\
3,127 \\
5,944 \\
19 \\
37\end{array}$ & $\begin{array}{r}6,831 \\
23,119 \\
33,431 \\
6,195 \\
3,831\end{array}$ \\
\hline $\begin{array}{l}\text { Rutherford } \\
\text { Sampson } \\
\text { Scotland } \\
\text { Stanly } \\
\text { Stokes }\end{array}$ & $\begin{array}{r}26,285 \\
27,407 \\
6,704 \\
8,387 \\
1,164\end{array}$ & $\begin{array}{r}7,697 \\
3,314 \\
1,124 \\
37 \\
--\end{array}$ & $\begin{array}{r}33,982 \\
30,721 \\
7,828 \\
8,424 \\
1,164\end{array}$ \\
\hline $\begin{array}{l}\text { Surry } \\
\text { Swain } \\
\text { Transylvania } \\
\text { Tyrrell } \\
\text { Union }\end{array}$ & $\begin{array}{r}36,835 \\
7,338 \\
4,272 \\
15,000 \\
15,496\end{array}$ & $\begin{array}{r}-- \\
2,751 \\
14,084 \\
-- \\
604\end{array}$ & $\begin{array}{l}36,835 \\
10,089 \\
18,356 \\
15,000 \\
16,100\end{array}$ \\
\hline $\begin{array}{l}\text { Vance } \\
\text { Wake } \\
\text { Warren } \\
\text { Washington } \\
\text { Watauga }\end{array}$ & $\begin{array}{r}7,391 \\
32,866 \\
26,064 \\
8,000 \\
=-\end{array}$ & $\begin{array}{r}251 \\
2,603 \\
1,133 \\
1,278 \\
--\end{array}$ & $\begin{array}{r}7,642 \\
35,469 \\
27,197 \\
9,278 \\
-\end{array}$ \\
\hline $\begin{array}{l}\text { Wayne } \\
\text { Wilkes } \\
\text { Wilson } \\
\text { Yadkin } \\
\text { Yancey }\end{array}$ & $\begin{array}{r}7,065 \\
11,208 \\
8,304 \\
-- \\
335\end{array}$ & $\begin{array}{r}1,340 \\
13 \\
1,131 \\
- \\
726\end{array}$ & $\begin{array}{r}8,405 \\
11,221 \\
9,435 \\
1,061\end{array}$ \\
\hline Total & $1,437,096$ & 326,020 & $1,763,116$ \\
\hline
\end{tabular}

Table 12.--Round pulpwood production in Oklahoma, 1957

\begin{tabular}{lrrrrr}
\multicolumn{5}{c}{ County } & (In standard cords) \\
\hline Adair & Pine & $\vdots$ & Hardwood & $\vdots$ & Total \\
Atoka & 592 & -- & 592 \\
Choctaw & -- & -- & -- \\
Haskell & 1,805 & -- & 1,805 \\
Latimer & 951 & -- & 951 \\
Ie Flore & 3,424 & -- & 3,424 \\
McCurtain & 14,537 & -- & 14,537 \\
Mayes & 23,752 & 244 & 23,996 \\
Pushmataha & 6,311 & 7,809 & 7,809 \\
$\quad$ Total & 51,372 & -- & 6,311 \\
\hline
\end{tabular}


Table 13.--Kound pulpwood production in South Carolina, 1957

\begin{tabular}{|c|c|c|c|}
\hline County & Pine & Hardwood & Total \\
\hline $\begin{array}{l}\text { Abbeville } \\
\text { Aiken } \\
\text { Allendale } \\
\text { Anderson } \\
\text { Bamberg }\end{array}$ & $\begin{array}{r}34,506 \\
28,906 \\
17,228 \\
47,212 \\
7,195\end{array}$ & $\begin{array}{r}177 \\
315 \\
4,974 \\
64 \\
5,082\end{array}$ & $\begin{array}{l}34,683 \\
29,221 \\
22,202 \\
47,276 \\
12,277\end{array}$ \\
\hline $\begin{array}{l}\text { Barnwell } \\
\text { Beaufort } \\
\text { Berkeley } \\
\text { Calhoun } \\
\text { Charleston }\end{array}$ & $\begin{array}{r}6,980 \\
17,108 \\
35,546 \\
7,929 \\
39,899\end{array}$ & $\begin{array}{r}4,660 \\
3,782 \\
15,371 \\
886 \\
15,553\end{array}$ & $\begin{array}{r}11,640 \\
20,890 \\
50,917 \\
8,815 \\
55,452\end{array}$ \\
\hline $\begin{array}{l}\text { Cherokee } \\
\text { Chester } \\
\text { Chesterfleld } \\
\text { Clarendon } \\
\text { Colleton }\end{array}$ & $\begin{array}{l}16,384 \\
52,151 \\
16,118 \\
17,149 \\
43,931\end{array}$ & $\begin{array}{r}-- \\
2,554 \\
16,322 \\
2,655 \\
13,783\end{array}$ & $\begin{array}{l}16,384 \\
54,705 \\
32,440 \\
19,801+ \\
57,714\end{array}$ \\
\hline $\begin{array}{l}\text { Darlington } \\
\text { Dillon } \\
\text { Dorchester } \\
\text { Edgefield } \\
\text { Fairfield }\end{array}$ & $\begin{array}{r}17,774 \\
13,476 \\
30,734 \\
36,920 \\
103,856\end{array}$ & $\begin{array}{r}14,318 \\
2,715 \\
15,674 \\
3,871 \\
4,171\end{array}$ & $\begin{array}{r}32,09 ? \\
16,19] \\
46,408 \\
40,791 \\
108,027\end{array}$ \\
\hline $\begin{array}{l}\text { Florence } \\
\text { Georgetown } \\
\text { Greenville } \\
\text { Greenwood } \\
\text { Hampton }\end{array}$ & $\begin{array}{l}28,730 \\
86,752 \\
20,538 \\
41,516 \\
30,927\end{array}$ & $\begin{array}{r}5,650 \\
35,560 \\
329 \\
653 \\
10,543\end{array}$ & $\begin{array}{r}34,380 \\
122,312 \\
20,867 \\
42,169 \\
41,470\end{array}$ \\
\hline $\begin{array}{l}\text { Horry } \\
\text { Jasper } \\
\text { Kershaw } \\
\text { Lancaster } \\
\text { Laurens }\end{array}$ & $\begin{array}{l}27,738 \\
20,041 \\
38,875 \\
39,527 \\
41,621\end{array}$ & $\begin{array}{r}1,377 \\
5,395 \\
12,826 \\
5,770 \\
545\end{array}$ & $\begin{array}{l}29,115 \\
25,436 \\
51,701 \\
45,297 \\
42,166\end{array}$ \\
\hline $\begin{array}{l}\text { Lee } \\
\text { Lexington } \\
\text { MeCormick } \\
\text { Marion } \\
\text { Marlboro }\end{array}$ & $\begin{array}{r}10,386 \\
14,438 \\
30,242 \\
10,989 \\
7,185\end{array}$ & $\begin{array}{r}6,083 \\
1,077 \\
\ldots \\
3,378 \\
6,112\end{array}$ & $\begin{array}{l}16,469 \\
15,515 \\
30,242 \\
14,367 \\
13,297\end{array}$ \\
\hline $\begin{array}{l}\text { Newberry } \\
\text { Oconee } \\
\text { Orangeburg } \\
\text { P1ckens } \\
\text { Richlend }\end{array}$ & $\begin{array}{r}68,801 \\
31,002 \\
24,107 \\
8,963 \\
37,145\end{array}$ & $\begin{array}{r}12,196 \\
1,223 \\
8,594 \\
856 \\
14,102\end{array}$ & $\begin{array}{r}80,997 \\
32,225 \\
32,701 \\
9,819 \\
51,247\end{array}$ \\
\hline $\begin{array}{l}\text { Saluda } \\
\text { Spartanburg } \\
\text { Sumter } \\
\text { Union } \\
\text { W111 iamsburg } \\
\text { York }\end{array}$ & $\begin{array}{l}15,895 \\
39,806 \\
30,778 \\
39,639 \\
11,162 \\
36,862\end{array}$ & $\begin{array}{r}1,256 \\
5,060 \\
1,280 \\
2,856 \\
689\end{array}$ & $\begin{array}{l}15,895 \\
41,062 \\
35,838 \\
40,919 \\
14,018 \\
37,551\end{array}$ \\
\hline Total & $1,384,667$ & 270,337 & $1,655,004$ \\
\hline
\end{tabular}

Table 14.--Round pulpwood production in Tennessee, 1957

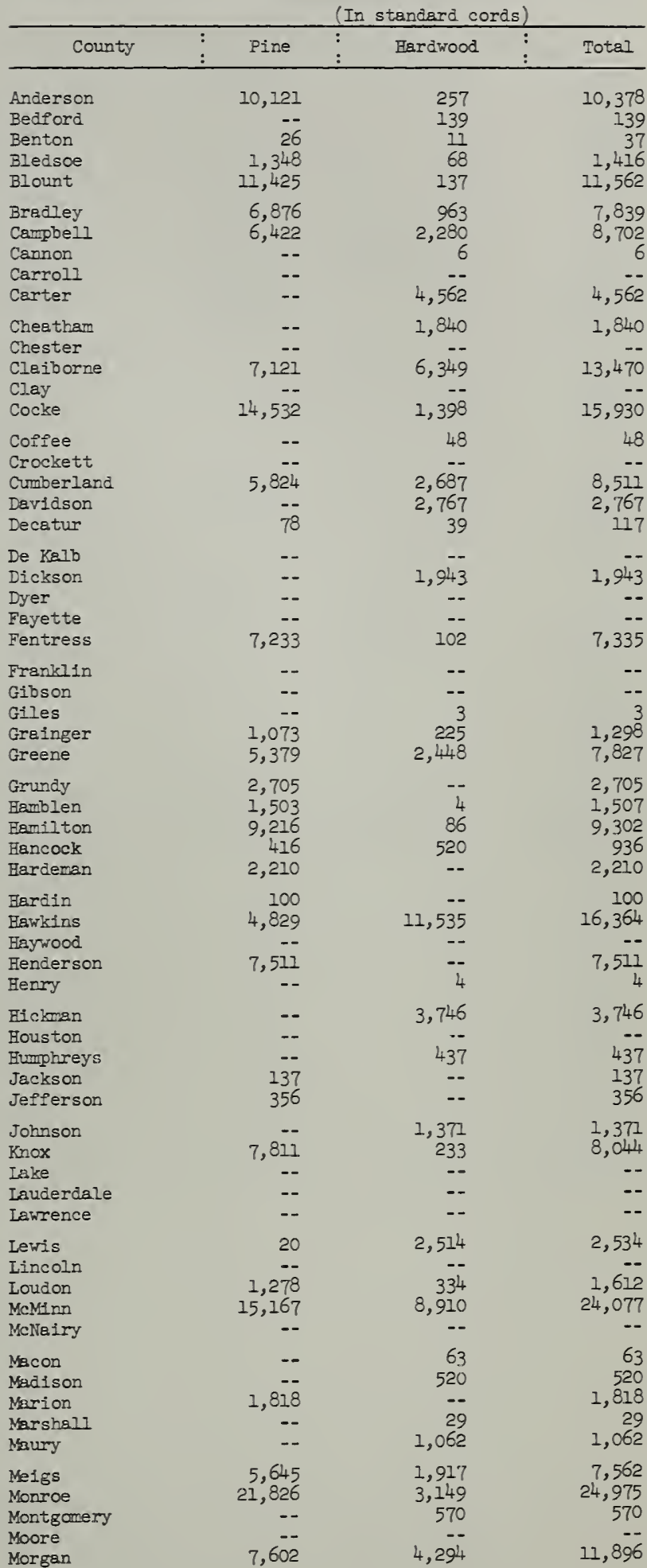


Table 14.--Round pulpwood production in Tennessee, 1957 (continued)

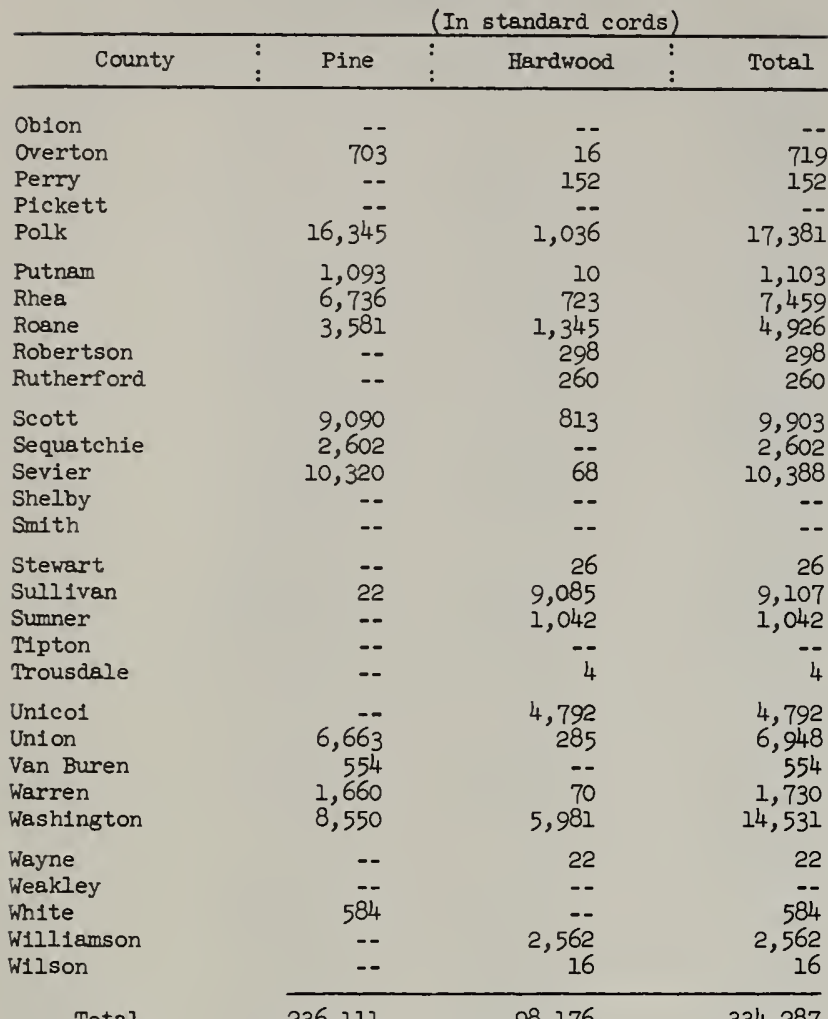

Table 15.--Round pulpwood production in Texas, 1957

\begin{tabular}{|c|c|c|c|}
\hline County & Plne & Hardwood & Total \\
\hline $\begin{array}{l}\text { Anderson } \\
\text { Angelina } \\
\text { Austin } \\
\text { Bastrop } \\
\text { Bowie }\end{array}$ & $\begin{array}{r}12,523 \\
44,019 \\
-- \\
546 \\
15,561\end{array}$ & $\begin{array}{r}3,100 \\
217 \\
1,073 \\
-- \\
--\end{array}$ & $\begin{array}{r}15,623 \\
44,236 \\
1,073 \\
546 \\
15,561\end{array}$ \\
\hline $\begin{array}{l}\text { Brazos } \\
\text { Burlesor } \\
\text { Caldwell } \\
\text { Camp } \\
\text { Cass }\end{array}$ & $\begin{array}{r}-- \\
-- \\
4,668 \\
33,383\end{array}$ & $\begin{array}{r}753 \\
753 \\
-- \\
53 \\
644\end{array}$ & $\begin{array}{r}753 \\
753 \\
-- \\
4,721 \\
34,027\end{array}$ \\
\hline $\begin{array}{l}\text { Chambers } \\
\text { Cherokee } \\
\text { Childress } \\
\text { Colorado } \\
\text { Fayette }\end{array}$ & $\begin{array}{r}-- \\
42,577 \\
314 \\
391 \\
--\end{array}$ & $\begin{array}{r}-- \\
2,752 \\
3 \\
234 \\
--\end{array}$ & $\begin{array}{r}-- \\
45,329 \\
317 \\
625 \\
--\end{array}$ \\
\hline $\begin{array}{l}\text { Fort Bend } \\
\text { Franklin } \\
\text { Caines } \\
\text { Gregg } \\
\text { Grimes }\end{array}$ & $\begin{array}{r}1,583 \\
12,011 \\
12,360\end{array}$ & $\begin{array}{r}137 \\
-- \\
- \\
744 \\
3,484\end{array}$ & $\begin{array}{r}137 \\
1,583 \\
12,755 \\
15,844\end{array}$ \\
\hline $\begin{array}{l}\text { Hall } \\
\text { Hardin } \\
\text { Harris } \\
\text { Harrison } \\
\text { Henderson }\end{array}$ & $\begin{array}{r}20 \\
31,885 \\
27,847 \\
40,036 \\
2,321\end{array}$ & $\begin{array}{r}10,417 \\
16,709 \\
1,842 \\
--\end{array}$ & $\begin{array}{r}20 \\
42,302 \\
44,556 \\
41,878 \\
2,321\end{array}$ \\
\hline $\begin{array}{l}\text { Hopkins } \\
\text { Houston } \\
\text { Jasper } \\
\text { Jefferson } \\
\text { Johnson }\end{array}$ & $\begin{array}{r}3 \\
49,452 \\
55,629 \\
2,243 \\
12\end{array}$ & $\begin{array}{r}-- \\
2,928 \\
13,520 \\
771 \\
--\end{array}$ & $\begin{array}{r}3 \\
52,380 \\
69,149 \\
3,014 \\
12\end{array}$ \\
\hline $\begin{array}{l}\text { Lamar } \\
\text { Lee } \\
\text { Leon } \\
\text { Liberty } \\
\text { Madison }\end{array}$ & $\begin{array}{r}39 \\
-- \\
868 \\
47,114 \\
74\end{array}$ & $\begin{array}{r}-- \\
-- \\
-- \\
10,573 \\
--\end{array}$ & $\begin{array}{r}39 \\
-- \\
868 \\
57,687 \\
74\end{array}$ \\
\hline $\begin{array}{l}\text { Marion } \\
\text { Montgomery } \\
\text { Morris } \\
\text { Nacogdoches } \\
\text { Newton }\end{array}$ & $\begin{array}{r}22,390 \\
60,944 \\
6,857 \\
72,509 \\
36,021\end{array}$ & $\begin{array}{r}2,513 \\
14,883 \\
=- \\
1,069 \\
10,220\end{array}$ & $\begin{array}{r}24,903 \\
75,827 \\
6,857 \\
73,578 \\
46,241\end{array}$ \\
\hline $\begin{array}{l}\text { Orange } \\
\text { Panola } \\
\text { Polk } \\
\text { Red River } \\
\text { Rusk }\end{array}$ & $\begin{array}{r}9,510 \\
33,536 \\
39,909 \\
2,525 \\
39,002\end{array}$ & $\begin{array}{r}408 \\
1,201 \\
12,161 \\
1,762\end{array}$ & $\begin{array}{r}9,918 \\
34,737 \\
52,070 \\
2,525 \\
40,764\end{array}$ \\
\hline $\begin{array}{l}\text { Sabine } \\
\text { San Augustine } \\
\text { San Jacinto } \\
\text { Shelby } \\
\text { Smith }\end{array}$ & $\begin{array}{l}33,499 \\
42,008 \\
11,076 \\
52,467 \\
16,873\end{array}$ & $\begin{array}{l}4,163 \\
8,406 \\
3,258 \\
4,635 \\
1,984\end{array}$ & $\begin{array}{l}37,662 \\
50,414 \\
14,334 \\
57,102 \\
18,857\end{array}$ \\
\hline $\begin{array}{l}\text { Terry } \\
\text { Titus } \\
\text { Trinity } \\
\text { Tyler } \\
\text { Upshur }\end{array}$ & $\begin{array}{r}1,109 \\
39,974 \\
38,411 \\
26,690\end{array}$ & $\begin{array}{r}-- \\
2,576 \\
8,997 \\
1,421\end{array}$ & $\begin{array}{r}1,109 \\
42,550 \\
47,408 \\
28,111\end{array}$ \\
\hline $\begin{array}{l}\text { Van Zandt } \\
\text { Walker } \\
\text { Waller } \\
\text { Wharton } \\
\text { Wood }\end{array}$ & $\begin{array}{r}36,084 \\
40 \\
-- \\
6,777\end{array}$ & $\begin{array}{r}1,648 \\
7,366 \\
175 \\
975 \\
-- \\
\end{array}$ & $\begin{array}{r}1,648 \\
43,450 \\
215 \\
975 \\
6,777 \\
\end{array}$ \\
\hline Total & $1,065,690$ & 160,528 & $1,226,21.8$ \\
\hline
\end{tabular}


Table 16.--Round pulpwood production in Virginia, 1957

\begin{tabular}{|c|c|c|c|}
\hline County & Pine & $: \quad$ Hardwood & Total \\
\hline $\begin{array}{l}\text { Accomack } \\
\text { Albemarle } \\
\text { Alleghany } \\
\text { Amelia } \\
\text { Amherst }\end{array}$ & $\begin{array}{r}5,608 \\
26,450 \\
17,749 \\
17,371 \\
30,008\end{array}$ & $\begin{array}{r}4,142 \\
71,399 \\
1,986 \\
30,180\end{array}$ & $\begin{array}{r}5,608 \\
30,592 \\
89,148 \\
19,357 \\
60,188\end{array}$ \\
\hline $\begin{array}{l}\text { Appomattox } \\
\text { Arlington } \\
\text { Augusta } \\
\text { Bath } \\
\text { Bedford }\end{array}$ & $\begin{array}{r}24,804 \\
7 \\
7,308 \\
2,499 \\
39,195\end{array}$ & $\begin{array}{r}9,269 \\
\ldots \\
3,875 \\
21,697 \\
13,751\end{array}$ & $\begin{array}{r}34,073 \\
7 \\
11,183 \\
24,196 \\
52,946\end{array}$ \\
\hline $\begin{array}{l}\text { Bland } \\
\text { Botetourt } \\
\text { Brunswick } \\
\text { Buchanan } \\
\text { Buckingham }\end{array}$ & $\begin{array}{r}5 \\
7,220 \\
36,269 \\
-2 \\
40,586\end{array}$ & $\begin{array}{r}11,044 \\
5,704 \\
\cdots \\
24,031\end{array}$ & $\begin{array}{r}5 \\
18,264 \\
41,973 \\
64,617\end{array}$ \\
\hline $\begin{array}{l}\text { Campbell } \\
\text { Caroline } \\
\text { Carroll } \\
\text { Charles City } \\
\text { Charlotte }\end{array}$ & $\begin{array}{r}39,353 \\
21,103 \\
78 \\
11,041 \\
24,569\end{array}$ & $\begin{array}{r}7,367 \\
281 \\
-- \\
1,947 \\
1,611\end{array}$ & $\begin{array}{r}46,720 \\
21,384 \\
78 \\
12,988 \\
26,180\end{array}$ \\
\hline $\begin{array}{l}\text { Chesterfield } \\
\text { Clarke } \\
\text { Craig } \\
\text { Culpeper } \\
\text { Cumberland }\end{array}$ & $\begin{array}{r}14,203 \\
178 \\
4,073 \\
6,262 \\
22,523\end{array}$ & $\begin{array}{r}4,166 \\
-- \\
5,780 \\
4,356 \\
2,027\end{array}$ & $\begin{array}{r}18,369 \\
178 \\
9,853 \\
10,618 \\
24,550\end{array}$ \\
\hline $\begin{array}{l}\text { Dickenson } \\
\text { Dinwiddie } \\
\text { Elizabeth City } \\
\text { Essex } \\
\text { Fairfax }\end{array}$ & $\begin{array}{r}21,571 \\
5 \\
6,543 \\
4,606\end{array}$ & $\begin{array}{r}4,984 \\
-- \\
344 \\
333\end{array}$ & $\begin{array}{r}26,555 \\
5 \\
6,887 \\
4,939\end{array}$ \\
\hline $\begin{array}{l}\text { Fauquier } \\
\text { Floya } \\
\text { Fluvanna } \\
\text { Franklin } \\
\text { Frederick }\end{array}$ & $\begin{array}{r}4,314 \\
292 \\
13,770 \\
27,869 \\
6,713\end{array}$ & $\begin{array}{r}131 \\
-- \\
3,307 \\
51 \\
973\end{array}$ & $\begin{array}{r}4,4145 \\
292 \\
17,077 \\
27,920 \\
7,686\end{array}$ \\
\hline $\begin{array}{l}\text { Giles } \\
\text { Gloucester } \\
\text { Goochland } \\
\text { Grayson } \\
\text { Greene }\end{array}$ & $\begin{array}{r}7 \\
13,212 \\
24,355 \\
-- \\
3,851\end{array}$ & $\begin{array}{r}227 \\
695 \\
1,566 \\
-- \\
16\end{array}$ & $\begin{array}{r}234 \\
13,907 \\
25,921 \\
-- \\
3,867\end{array}$ \\
\hline $\begin{array}{l}\text { Greensville } \\
\text { Halifax } \\
\text { Hanover } \\
\text { Henrico } \\
\text { Henry }\end{array}$ & $\begin{array}{r}23,240 \\
19,756 \\
3,214 \\
5,149 \\
26,062\end{array}$ & $\begin{array}{r}2,378 \\
463 \\
480 \\
147 \\
--\end{array}$ & $\begin{array}{r}25,618 \\
20,219 \\
3,694 \\
5,296 \\
26,062\end{array}$ \\
\hline $\begin{array}{l}\text { Highland } \\
\text { Isle of Wight } \\
\text { James City } \\
\text { King and Queen } \\
\text { King George }\end{array}$ & $\begin{array}{r}249 \\
6,247 \\
9,250 \\
22,313 \\
825\end{array}$ & $\begin{array}{r}5,156 \\
2,405 \\
487 \\
1,174 \\
328\end{array}$ & $\begin{array}{r}5,405 \\
8,652 \\
9,737 \\
23,487 \\
1,153\end{array}$ \\
\hline $\begin{array}{l}\text { King William } \\
\text { Lancaster } \\
\text { Iee } \\
\text { I.oudoun } \\
\text { Louisa }\end{array}$ & $\begin{array}{r}17,566 \\
9,477 \\
-- \\
971 \\
22,537\end{array}$ & $\begin{array}{r}923 \\
-- \\
839 \\
84 \\
788\end{array}$ & $\begin{array}{r}18,489 \\
9,477 \\
839 \\
1,055 \\
23,325\end{array}$ \\
\hline
\end{tabular}

Table 16.--Round pulpwood production in Virginia, 1957 (continued

\begin{tabular}{|c|c|c|c|}
\hline County & Pine & $: \quad$ Hardwood & Total \\
\hline $\begin{array}{l}\text { Lunenburg } \\
\text { Madison } \\
\text { Mathews } \\
\text { Mecklenburg } \\
\text { Middlesex }\end{array}$ & $\begin{array}{r}14,707 \\
3,699 \\
1,650 \\
16,807 \\
7,886\end{array}$ & $\begin{array}{r}1,390 \\
43 \\
869 \\
1,356 \\
415\end{array}$ & $\begin{array}{r}16,097 \\
3,742 \\
2,519 \\
18,163 \\
8,301\end{array}$ \\
\hline $\begin{array}{l}\text { Montgomery } \\
\text { Nansemond } \\
\text { Nelson } \\
\text { New Kent } \\
\text { Norfolk }\end{array}$ & $\begin{array}{r}4,940 \\
4,609 \\
15,310 \\
15,439 \\
4,215\end{array}$ & $\begin{array}{r}3,335 \\
3,786 \\
1,023 \\
458\end{array}$ & $\begin{array}{r}4,940 \\
7,944 \\
19,096 \\
16,462 \\
4,673\end{array}$ \\
\hline $\begin{array}{l}\text { Northampton } \\
\text { Northumberland } \\
\text { Nottoway } \\
\text { Orange } \\
\text { Page }\end{array}$ & $\begin{array}{r}3,819 \\
17,635 \\
20,004 \\
975\end{array}$ & $\begin{array}{r}-- \\
3,956 \\
3,017 \\
40\end{array}$ & $\begin{array}{r}3,819 \\
21,591 \\
23,021 \\
1,015\end{array}$ \\
\hline $\begin{array}{l}\text { Patrick } \\
\text { Pittsylvania } \\
\text { Powhatan } \\
\text { Prince Edward } \\
\text { Prince George }\end{array}$ & $\begin{array}{l}2,561 \\
40,797 \\
14,978 \\
26,129 \\
14,827\end{array}$ & $\begin{array}{r}1,-- \\
7168 \\
2,660 \\
2,389\end{array}$ & $\begin{array}{r}2,561 \\
41,965 \\
15,694 \\
28,789 \\
17,216\end{array}$ \\
\hline $\begin{array}{l}\text { Prince William } \\
\text { Princess Anne } \\
\text { Pulaski } \\
\text { Rappahannock } \\
\text { Richmond }\end{array}$ & $\begin{array}{r}27,315 \\
13 \\
24 \\
-- \\
6,243\end{array}$ & $\begin{array}{c}218 \\
-- \\
-- \\
-- \\
--\end{array}$ & $\begin{array}{r}27,533 \\
13 \\
24 \\
-- \\
6,243\end{array}$ \\
\hline $\begin{array}{l}\text { Rosnoke } \\
\text { Rockbridge } \\
\text { Rockingham } \\
\text { Russe]1 } \\
\text { Scott }\end{array}$ & $\begin{array}{r}2,175 \\
9,781 \\
7,133 \\
-- \\
206\end{array}$ & $\begin{array}{r}19,622 \\
1,396 \\
426 \\
16,096\end{array}$ & $\begin{array}{r}2,175 \\
29,403 \\
8,529 \\
426 \\
16,302\end{array}$ \\
\hline $\begin{array}{l}\text { Shenandoah } \\
\text { Smyth } \\
\text { Southampton } \\
\text { Spotsylvania } \\
\text { Stafford }\end{array}$ & $\begin{array}{r}4,613 \\
-- \\
22,878 \\
14,809 \\
2,839\end{array}$ & $\begin{array}{r}86 \\
26 \\
8,201 \\
1,140 \\
331\end{array}$ & $\begin{array}{r}4,699 \\
26 \\
31,079 \\
15,949 \\
3,170\end{array}$ \\
\hline $\begin{array}{l}\text { Surry } \\
\text { Stissex } \\
\text { Tazewel]. } \\
\text { Warren } \\
\text { Warwick }\end{array}$ & $\begin{array}{r}10,597 \\
19,000 \\
-- \\
1,551 \\
1,404\end{array}$ & $\begin{array}{r}2,393 \\
3,681 \\
-- \\
295 \\
59\end{array}$ & $\begin{array}{r}12,990 \\
22,681 \\
-- \\
1,846 \\
1,463\end{array}$ \\
\hline $\begin{array}{l}\text { Washington } \\
\text { Westmoreland. } \\
\text { Wise } \\
\text { Wythe } \\
\text { York }\end{array}$ & $\begin{array}{r}4,116 \\
6,529 \\
-- \\
999 \\
14,543\end{array}$ & $\begin{array}{r}3,695 \\
-- \\
148 \\
7- \\
765\end{array}$ & $\begin{array}{r}7,811 \\
6,529 \\
148 \\
999 \\
15,308\end{array}$ \\
\hline Total & $1,080,181$ & 338,091 & $1,418,272$ \\
\hline
\end{tabular}




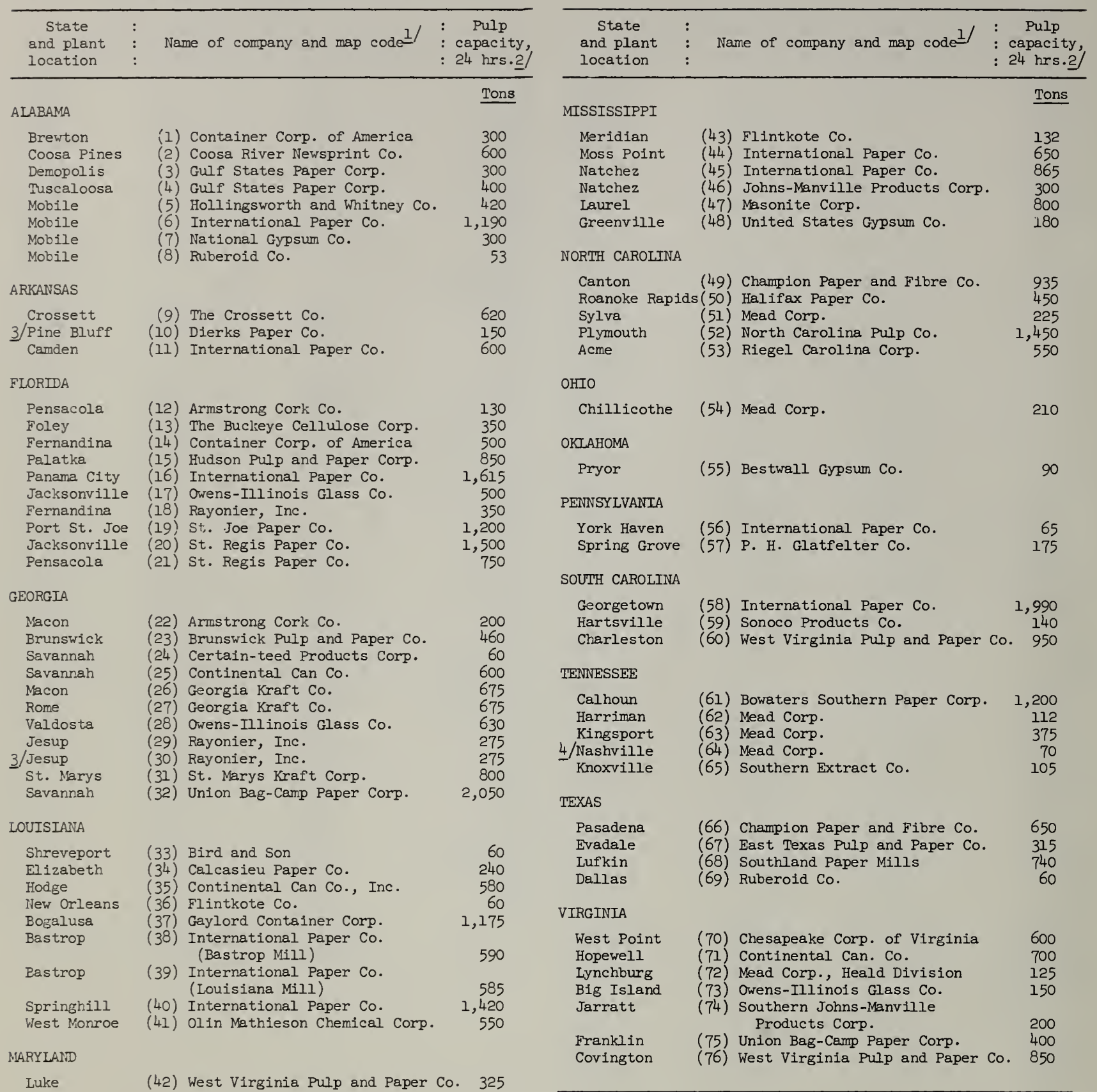

I) Corresponds to numbers at mill locations in figure 1 .

2) Southern Pulp and Paper Manufacturer, vol. 20, no. 10 (Oct. 1, 1957); and other sources.

3/ Started operation in January 1958.

4) Discontinued operation in March 1958. 
Table 18.--Pulpmills under construction or announced in the South

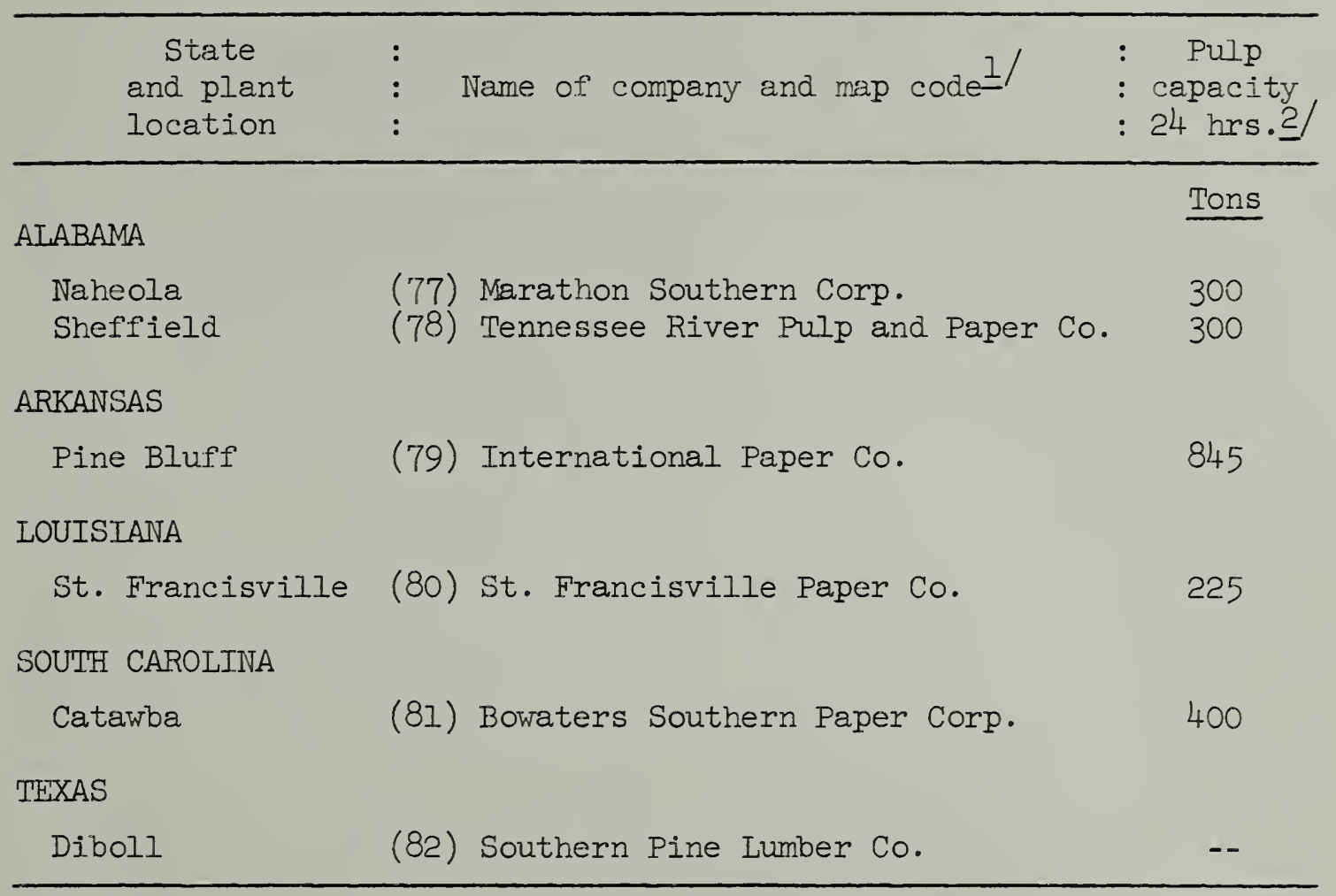

I/ Corresponds to numbers at mill location in figure 1 .

2/ Southern Pulp and Paper Manufacturer, and other sources. 



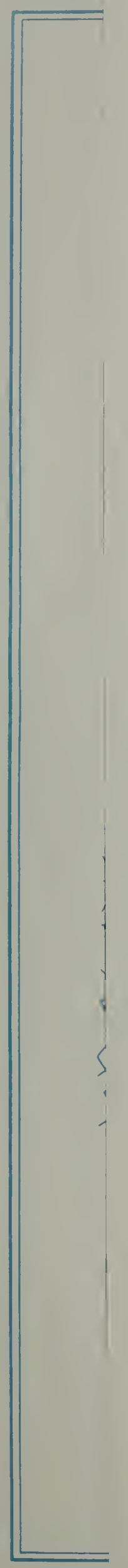





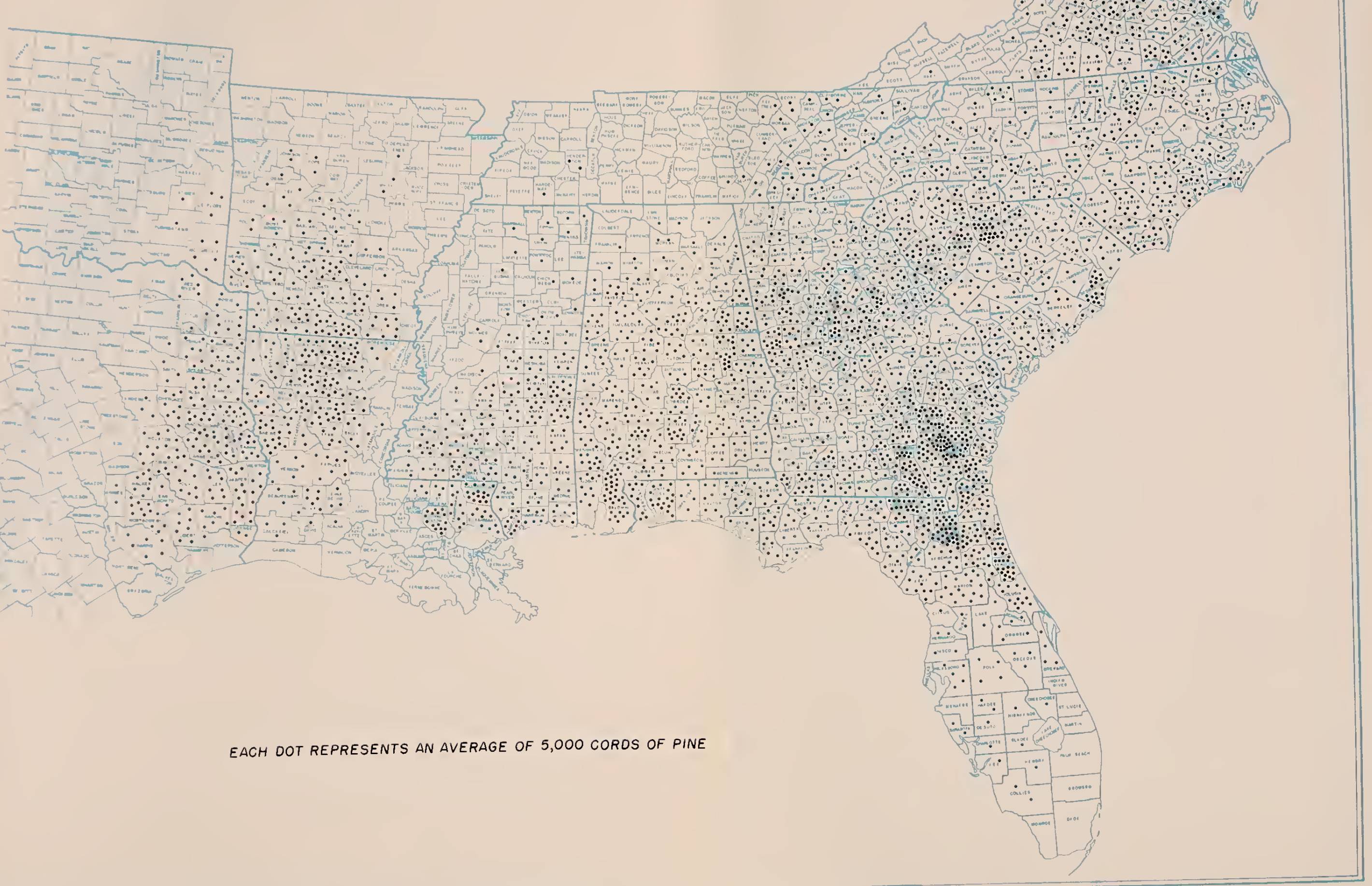

Figure 3.--Pine pulpwood production by county in the South, 1957. 



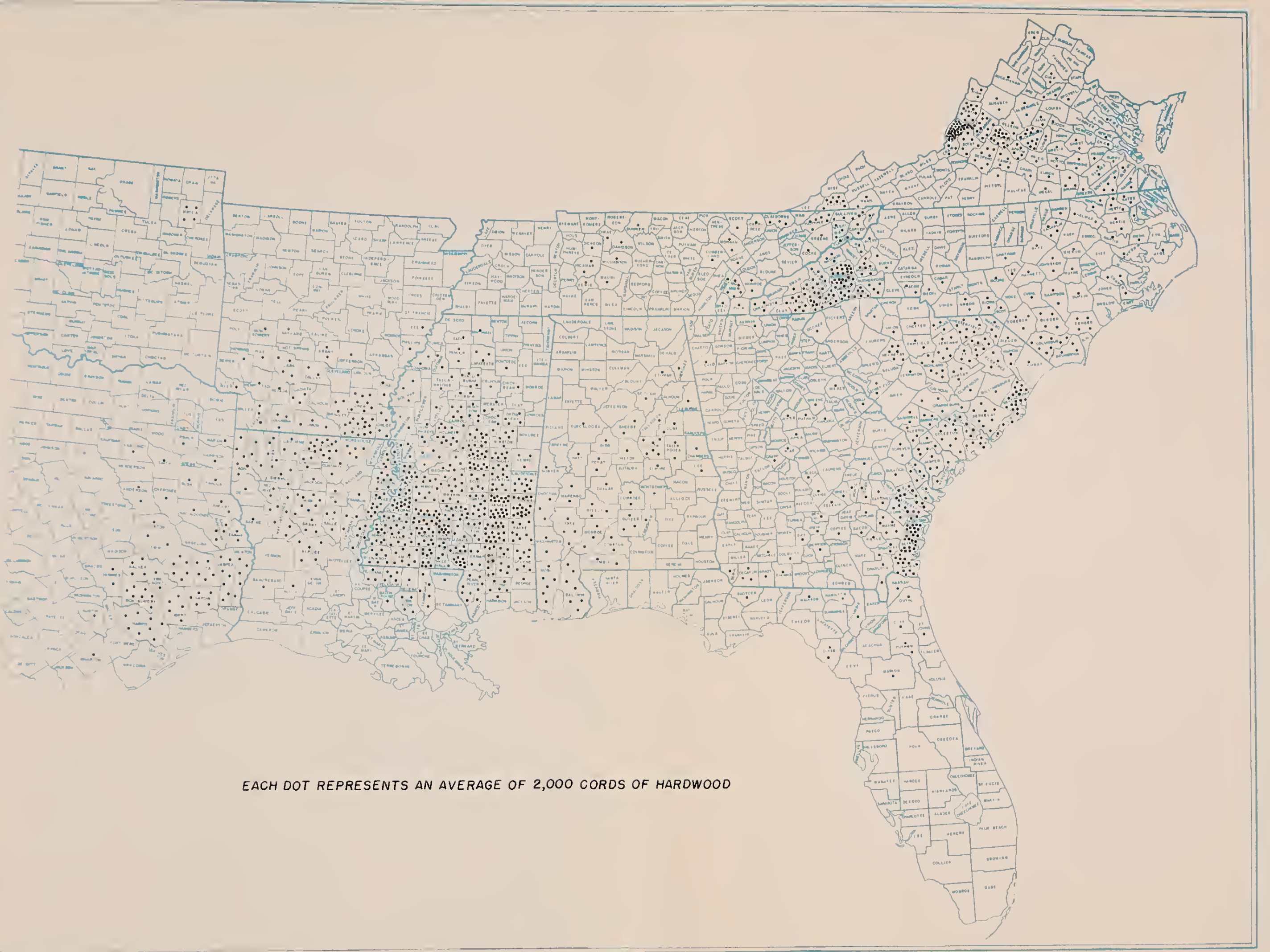

Figure 4.--Hardwood pulpwood production by county in the South, 1957. 


